\title{
Spheroid culture models imitating the tumor microenvironment of renal and melanoma cancer
}

\author{
Aleksandra Filipiak ( $\nabla$ afilipiak1@wim.mil.pl ) \\ Brodaczewska Klaudia \\ Military Institute of Medicine: Wojskowy Instytut Mediczny \\ Majewska Aleksandra \\ Military Institute of Medicine: Wojskowy Instytut Mediczny \\ Kieda Claudine \\ Military Institute of Medicine: Wojskowy Instytut Mediczny
}

Military Institute of Medicine: Wojskowy Instytut Mediczny https://orcid.org/0000-0002-8118-8468

Primary research

Keywords: EMT, CSC, hypoxia, 3D, RCC

Posted Date: February 14th, 2022

DOI: https://doi.org/10.21203/rs.3.rs-693762/v2

License: (9) This work is licensed under a Creative Commons Attribution 4.0 International License.

Read Full License 


\section{Abstract}

Tumor development studies should adapt to the cancer cells specific mechanisms in connection with their microenvironment. Standard two-dimensional (2D) cultures and gas composition are not relevant to the real cancer environment. Existing three-dimensional (3D) models are often requiring sophisticated conditions. Two cancer models were chosen: melanoma (B16F10) and kidney cancer (RenCa). We proposed and characterized a 3D culture method, reporting the presence of hypoxia-related genes/proteins and aggressiveness mechanism (EMT-Epithelial Mesenchymal Transition and CSC-Cancer Stem Cells). We validate the proposed 3D method by comparing it with in vivo obtained tumors. We designed method which brings simplicity and reproducibility of the data. Melanoma spheroid-growing cells reached a cell cycle arrest at the G0/G1 phase and showed induction of hypoxia. Spheroid-recovered RenCa cells were enriched in proliferating cells and displayed delayed hypoxia. A comparable response to hypoxia was observed in 3D spheroids and in vivo tumors for both lines. 3D induced CSC characters in RCC whereas EMT-related mechanisms occurred in the melanoma model. Such distinction in the use of separate aggressiveness-leading pathways was observed in in vivo melanoma vs kidney tumors. 3D models help mimic some features of the corresponding in vivo tumors such as hypoxia and aggressiveness mechanisms. We showed that these two cell models use distinct mechanisms to reach aggressiveness: epithelial to mesenchymal transition (EMT), cancer stem cells (CSC) by B16F10 and RenCa cells, respectively. These data on aggressiveness pathways and reaction to hypoxia confirm and validate the proposed 3D models for further design of alternative methods to in vivo studies.

\section{Introduction}

Despite advantages in cancer treatment research, this disease is still the leading cause of death worldwide (Bray et al., 2018). Treating and studies on cancers are challenging due to complexity of tumor site (Hinshaw and Shevde, 2019). This complex system is composed of cancer and many other noncancer cells, which all have specific role in the functioning of the tumor (Hoarau-Véchot et al., 2018). Along with the development of cancer cells the mass gets hypoxic and this conditions the development of a microenvironment of the tumor cells (Carreau et al., 2011). Such condition is defined as an oxygen tension lower than observed in non-pathologic conditions $\left(\mathrm{pO}_{2} 1 \%\right)$ (Sakashita et al., 2019). Hypoxia induces the expression of Hypoxia-inducible factor $1 \mathrm{a}(\mathrm{HIF-1} \mathrm{a})$. Transcription of HIF-1 in turn, induces angiogenic factors such as Vascular endothelial growth factor A (VEGF-A) (Abou Khouzam et al., 2020; Casanovas et al., 2005; Collet et al., 2012). This mechanism is controlled by the tumor suppressor von Hippel-Lindau (VHL) (J. Zhang and Zhang, 2018; Cowey and Rathmell, 2009; Rathmell and Chen, 2008; Schödel et al., 2016). In physiological oxygen tension, VHL binds to HIF-1a and promotes degradation of HIF-1a in proteasome (Khacho et al., 2008). In RCC cancers mutation of VHL gene is often occurring, which promotes a constant pathological hypoxic condition (Cowey and Rathmell, 2009; Rathmell and Chen, 2008). Hypoxia represents a poor prognosis factor in many cancers, it is linked with cancer aggressiveness, invasion, and metastasis (Joseph et al., 2018; Abou Khouzam et al., 2020; Carreau et al., 
2011). Epithelial to Mesenchymal Transition (EMT) process is one of the initiating steps of cancer invasion, during which tumor cells dedifferentiate by losing epithelial markers (e.g. E-cadherin) in favor of mesenchymal ones (e.g. N-cadherin, B-catenin, Vimentin) (Quail and Joyce, 2013). To study the biology of cancer cells in vitro, 3D cultures have been developed.

Metastasis process is also linked with the selection of highly aggressive cells - Cancer Stem Cells (CSC) (Melissaridou et al., 2019). These cells are characterized with stem-like features (Pan et al., 2018). It has been shown that CSCs are responsible for tumor initiation, progression, and disease relapse (Melissaridou et al., 2019; Vlashi and Pajonk, 2015). They are also thought to be responsible for the development of resistance to cancer treatment (Vlashi and Pajonk, 2015). CSCs could be found in melanoma and renal cancer, they were characterized with the expression of cell surface markers specific to the type of cancer, such as CD105 (Shi et al., 2019; Dolinsek et al., 2015), CD133 (Bradley et al., 2020; Simbulan-Rosenthal et al., 2019), Aldehyde dehydrogenase 1 (ALDH1) (Taylor et al., 2017; Abourbih et al., 2013).

Mimicking real cancer microenvironment is crucial in cancer research (Hinshaw and Shevde, 2019). Syngeneic in vivo murine models are highly valuable tools in terms of recreating the full spectrum of tumor (Cheon and Orsulic, 2011). However, animal models cannot always be used in each phase of preclinical studies. Therefore, most initial research is performed with the use of monolayer cell culture. Standard two-dimensional (2D) cell culture methods are highly limited, they are lacking the threedimensional shape of the tumor which largely modulate the cell to cell interactions and limits oxygen availability thus lowering oxygen tension (Duval et al., 2017). Hypoxic conditions can be reproduced in 2D cultures by usage of hypoxic chambers (Li et al., 2012), however the oxygen distribution does not reproduce the natural oxygen gradient as set in the tumor. Along with the spheroid development its 3D structure allows such natural establishment of hypoxia (Klimkiewicz et al., 2017). There are, already developed, different type 3D culture methods with a usage of scaffold and non-scaffold methods (Ravi et al., 2015). However, by various factors, e.g., cost, special equipment and reproducibility of the highly complicated methods, widespread implementation of 3D's into cell-based studies has been limited (Verjans et al., 2018; Ravi et al., 2015; Singh et al., 2020; Hoarau-Véchot et al., 2018). Commercially available products for the 3D cell culture (e.g. hanging drop or scaffold plates) are also not always well optimized and due to the lack of standardized protocols interpretation of the data can be challenging (Verjans et al., 2018)

The aim of the present studies was to optimize and validate simple in vitro models which will correspond to the characteristics observed in the in vivo tumor, such as: three-dimensional shape of the tumor, proper oxygen tension and cellular responses (Hoarau-Véchot et al., 2018; Quail and Joyce, 2013). We used two different cell lines to establish a universal protocol allowing sphere formation. We characterize the obtained 3D structures compared to standard monolayer culture with a special emphasis on EMT and CSC related markers. The goal of the study was to assess whether cells undergo similar molecular changes in spheroids as in the tumor mass in vivo. Such well-characterized models may contribute to 
obtaining more reliable data in an easy way, which will bring research tools to provide data closer to the possible translation into clinical practice.

\section{Animals, Material And Methods}

\section{Animals - In vivo tumor implantation and development}

The experiments were carried out with 12 female mice. Two biological repetitions, $6(2 \times 3)$ of the C57BI6 strain - syngeneic model of melanoma and two biological repetitions, 6 (2x3) of BALB /C - syngeneic model of kidney tumor. The mice had an acclimatization time before starting the experiments. During the experiment the mice were housed in Military Institute of Hygiene and Epidemiology animal facility (listed as an experimental unit authorized to carry out animal experiments by the polish Ministry of Science and Higher Education, user number 0074), in IVC rack system in single-use, ventilated, plastic cages (Allentown, USA). Animals were maintained in a 12h / 12h light-dark cycle (dawn: 6:30-7:00) at $21 \pm 2{ }^{\circ} \mathrm{C}$ temperature and a relative humidity of $60 \pm 20 \%$. The food and water were provided ad libitum. As enrichment the wooden tool and nesting material was supplied. Cancer cell suspensions: RenCa $-100,000$ cells; B16F10 - 200,000 cells in $100 \mu \mathrm{L}$ of PBS mixed with Matrigel ${ }^{\mathrm{TM}}(50 \%$, vol/vol) (Corning, USA) and maintained at $4[\mathrm{C}$, were subcutaneously administered to the animal's thigh after anesthesia with isoflurane. Experimental groups consisted of 3 mice kept in one cage for each experimental group. The renal cancer model experiment was terminated after 22 days, while the melanoma cancer model experiment was completed 18 days after the injection of the tumor cells, when the tumors exceeded $1 \mathrm{~cm}$ diameter. All experiments were conducted after obtaining approval for procedures from Second Warsaw Local Ethics Committee for Animal Experimentation (no. WAW2/76/2017) and following Directive 2010/63/EU regulations.

\section{Cell culture}

The tests were carried out with murine renal carcinoma RenCa (CRL-2947 ${ }^{\mathrm{T}}$, ATCC, USA) and melanoma B16-F10 (given by Prof. J. Dulak, Department of Medical Biotechnology, Faculty of Biochemistry, Biophysics and Biotechnology, Jagiellonian University, Cracow, Poland; authenticated in 2021, ATCC) cell lines. Cells were maintained at $37{ }^{\circ} \mathrm{C}$ in a humidified atmosphere containing $5 \% \mathrm{CO}_{2}$ in appropriate media - RPMI-1640 GlutaMaxTM medium (ThermoFisher Scientific, Waltham, Massachusetts, USA), with 10\% FBS ( $\mathrm{vol} / \mathrm{vol}$ ) (ThermoFisher Scientific). After confluence reached $70-80 \%$ all cell lines were passaged by detaching with Accutase solution (Biolegend, San Diego, California, USA). Cells used in the experiments were Mycoplasma free as assayed with PCR Mycoplasma Test (Biomedica, Poland) and did not exceed 15th passage.

\section{Two-dimensional cell culture}


Two-dimensional cultures were carried out under two oxygen partial pressures "normoxia" (18,75\% 02)2DN and hypoxia (1\% 02)- 2DH. Cells were seeded at distinct densities: RenCa - $6600 \mathrm{cells} / \mathrm{cm}^{2}, \mathrm{~B} 16 \mathrm{~F} 10$ $3300 \mathrm{cell} / \mathrm{s} / \mathrm{cm}^{2} .24$ hours after plating, hypoxia intended cell cultures were transferred to hypoxia chambers (BioSpherix, Xvivo System Model X3, USA), while changing the cell medium to fresh hypoxiabalanced medium. Normoxic cell cultures were kept under standard aerobic conditions, after switching to fresh, normoxic medium. The cultures were pursued for 72 hours.

\section{Three-dimensional cell cultures by spheroid formation}

500 cells resuspended in $20 \mu \mathrm{L}$ medium supplemented with $0.25 \%$ methylcellulose (vol/vol in medium), (R\&D Systems, USA) were seeded as a single hanging drop, on the cover of culture plate in standard culture conditions $\left(5 \% \mathrm{CO}_{2}, 21 \% \mathrm{pO}_{2}\right)$. After $72 \mathrm{~h}$ drops were individually transferred to a 96 -well plate previously covered with $1 \%$ agarose dissolved in double distilled water (w/vol, VWR, Belgium), and $50 \mu \mathrm{L}$ of fresh medium (supplemented with $0,25 \%$ methylcellulose, vol/vol) were added concomitantly. Cells were allowed to expand for another four days period.

\section{Western blot}

The two-dimensional cell cultures were washed twice with ice-cold PBS and collected by scraping. Harvested and PBS-washed spheroids were dispersed, and cell suspensions were centrifuged before lysing by RIPA lysis buffer complemented with proteases and phosphatases inhibitors (both from ThermoFisher Scientific, USA). Fragments of neoplastic tissues were homogenized using TissueLyser II (Qiagen) in RIPA lysis buffer with proteases/phosphatases inhibitors. Insoluble material was removed by lysates centrifugation $\left(14,000 \times \mathrm{g}\right.$ for $10 \mathrm{~min}$ at $\left.4^{\circ} \mathrm{C}\right)$. Equal amount of total protein was separated on $12 \%$ SDS polyacrylamide gels for 40 min at a constant $200 \mathrm{~V}$ using a Bio-Rad Minigel System, followed by transfer to nitrocellulose membranes (GE Healthcare life science, Germany). Membranes, after blocking, were probed with primary antibodies to detect: ALDH1A1 (1:250 Polyclonal; LSBio), VHL (1:200 anti-mo; Clone: FL-181; Santa Cruz Biotechnology), HIF1a (1:100; Clone: 28b; Santa Cruz Biotechnology), Vimentin (1:1000 anti-rb, clone: D21H3; Cell Signaling Technology) and Vinculin (1:1000 anti-mo, clone: V284; Santa Cruz Biotechnology), which served as loading control. Membranes were further treated with secondary antibodies conjugated with horseradish peroxidase-HRP (goat anti - mouse/anti-rabbit, 1: 10,000; Vector Laboratories, USA). Bands were detected using Luminol as HRP substrate (Santa-Cruz, USA) with X-ray films. Bands were analyzed using ImageJFiji. All data were normalized to 2DN condition.

\section{Flow cytometry detection of intracellular enzymes activity and cell surface antigens}

Cell suspensions from 2D cultured cells were obtained using Accutase solution (Biolegend, USA). Individually cultured spheroids were treated with $0,1 \mathrm{mg} / \mathrm{ml}$ collagenase type II (Gibco by Life Technologies) and incubated at $37^{\circ} \mathrm{C}$ with simultaneous stirring until the spheres disintegrated into single cells.

\section{Aldehyde dehydrogenase- 1 activity assessment}


The ALDH1 enzyme activity was measured intracellularly with the help of Aldefluor fluorescent reagent. It is a BODIPY-amino acetaldehyde fluorescent ALDH substrate able to diffuse into intact and viable cells. Converted into BODIPY-amino-acetate by ALDH in cells, the negatively charged product is retained inside the cells increasing fluorescence for flow cytometry analysis ( $\lambda$ ex $\max =493 \mathrm{~nm}, \lambda$ em $\max =588 \mathrm{~nm}$ ). Protocol was followed according to the manufacturer's recommendations (STEMCELL Technologies). Briefly, $0.5 \times 10^{6}$ cells were suspended in $500 \mu \mathrm{L}$ of Aldefluor assay buffer. Assay samples received 2,5 $\mu \mathrm{l}$ of the-Aldefluor reagent, mixed and immediately $250 \mu \mathrm{l}$ of the suspension was taken out and put in the control tube with the DEAB ( $N, N^{\prime}$-dimethylaminobenzaldehyde) inhibitor 2,5 $\mu \mathrm{L}$. Cells were further incubated for 45 minutes at $37^{\circ} \mathrm{C}$ in the dark. After washing cells, viability was determined by staining with 7-Amino-Actinomycin $\mathrm{D}$ (BD Biosciences) a membrane permeant dye which is excluded from viable cells, it binds to double stranded DNA of dead cells ( $\lambda$ ex $\max =546 \mathrm{~nm}, \lambda$ em max $=647 \mathrm{~nm}$ ). All samples were analyzed using a Calibur flow cytometer (from Becton Dickinson, Sunnyvale Ca, USA). Cells treated with inhibitor were used to set the gate for ALDHneg population and positive cells were analyzed. All data were normalized to $2 \mathrm{DN}$ condition.

\section{Surface antigens detection by fluorescent antibodies}

The evaluation of the expression of CD105 and CD133 was determined using commercially available antibodies: PerCP-eFluor710 conjugate rat-anti-mo (IgG1) CD133 (Clone: 13A4; Thermo Fisher Scientific, USA) and PE conjugate rat-anti-mo (IgG2a) CD105 (Clone: MJ7/18; Thermo Fisher Scientific, USA). 500,000 cells were resuspended in PBS $(200 \mu \mathrm{l})$ and first stained for viability determination by LIVE/DEAD ${ }^{\text {TM }}$ Fixable Dead Cell Stain Sampler Kit, $488 \mathrm{~nm}$ excitation (Thermo Fisher Scientific, USA) for fluorescence spectra compatibility (for $15 \mathrm{~min}$ ). The dye reacts with free amines both inside and on the

surface of dead cells, yielding to an intense fluorescent staining. The cells were washed and resuspended in Stain Buffer - BSA (BD Pharmingen), then the labelling for antigens was performed by incubation of the cells in the presence of fluorescent antibodies for $2 \mathrm{~h}$ at $4{ }^{\circ} \mathrm{C}$ in the dark. Cell analysis was performed using Calibur flow cytometer (Becton and Dickinson, USA). All data were normalized to 2DN condition.

\section{Reverse Transcriptase-Polymerase Chain Reaction}

RNA samples from in vitro and in vivo cultures were isolated using the commercially available RNeasy Mini Kit (Qiagen, Hilden, Germany), according to the manufacture protocol. The obtained cell lysates were either stored at $-80^{\circ} \mathrm{C}$ or RNA isolation was started. The isolated RNA was then purified using the TURBOTM DNA-free Kit (Thermo Fisher Scientific, USA) according to the manufacturer's protocol. The concentration and purity of RNA were determined by measuring the absorbance in the wavelength range: 230, 260 and $280 \mathrm{~nm}$. RNA integrity was assessed using a fluorometer based on the RIN (RNA integrity number) coefficient (acceptable values 7-9). After obtaining high purity mRNA, a reverse transcription process, using a commercially available High-Capacity cDNA Reverse Transcription Kit (Thermo Fisher Scientific, USA) was performed according to the manufacturer's instructions. The resulting singlestranded cDNA was used as real-time polymerase chain reaction templates. 
RT-PCR reactions were performed using TaqMan Gene Expression Master Mix (Thermo Fisher Scientific, USA) together with commercially available probes all from Thermo Fisher Scientific: Vhl: Mm00494137, Vegf-a: Mm00437306, Hif-1a Mm00468869, Actin $\beta$ Mm02619580. The samples run on Bio-Rad CFX384 qPCR System or CFX Connect qPCR System (Bio-Rad). Amplification conditions were as follows: $50^{\circ} \mathrm{C}(2$ $\mathrm{min}), 95^{\circ} \mathrm{C}(10 \mathrm{~min})$ and 40 cycles of $95^{\circ} \mathrm{C}(15 \mathrm{~s})$ and $60^{\circ} \mathrm{C}(1 \mathrm{~min})$. The quantity of mRNA was calculated using the 2 (-Delta $\mathrm{C}(\mathrm{T})$ ) method and normalized to $\beta$-actin. All reactions were performed as triplicates. All data were normalized to $2 \mathrm{DN}$ condition.

\section{Next Generation Sequencing (NGS)}

NGS was performed outsourced service by Lexogen GmbH (Vienna, Austria) with NextSeq 500 system (Illumina, USA). Briefly, RNA was extracted and purified using methods described in Reverse

Transcriptase-Polymerase Chain Reaction section. RNA BR Assay Kit (Thermo Fisher Scientific, USA) was used to measure total RNA concentration and Qubit RNA IQ Assay Kit (Thermo Fisher Scientific, USA) to measure RNA integrity and quality. To obtain cDNA libraries $1 \mu \mathrm{g}$ of total RNA with IQ $>8,5$ was used.

Preparation of libraries was performed according to manufactures instructions as followed: The NEBNext ${ }^{\circledR}$ Poly $(A)$ mRNA Magnetic Isolation Module - mRNA isolation and fragmentation (New England Biolabs) Ultra RNA Library Prep Kit for Illumina - cDNA Library synthesis, New England Biolabs); NEBNext Multiplex Oligos for llumina - adding Adaptors (New England Biolabs). All steps were performed along with the purification of the reaction products using NEBNext Sample purification Beads (New England Biolabs). Libraries were qualified on Bioanalyzer (Agilent, USA), with High Sensitivity DNA Kit (Agilent, USA). Differentially expressed genes (DEGs) were classified according to $-1,5<\log F C>1,5$ and $p$-value $<$ 0.5. Gene symbols were translated into UniProt accession numbers using the UniProt Knowledgebase (UniProtKB). Protein networks were retrieved from the STRING (Search Tool for the Retrieval of Interacting Genes) database using the list of protein accession numbers as a query and then analyzed using the Cytoscape software.

\section{Microscopy Imaging}

$3 \mathrm{D}$ cultures were performed according to the above-mentioned procedure. Imaging to assess live and dead cells in the spheres was performed after labelling by calcein acetoxymethyl ester (Calcein AM, Biolegend) which is hydrolyzed by cellular esterases to give fluorescent calcein in the cytoplasm of live cells and propidium iodide (Exbio, Czech Republic) DNA intercalating agent which is highly fluorescent after intercalation. Briefly, after adding the dyes spheroids and cells grown in two-dimensions were incubated for 15 minutes in the dark at room temperature. Images were acquired using a Zeiss AxioObserver.7, fluorescence, and inverted microscope (5X magnification) and analysis performed with the Zen 2.6 blue edition software (Zeiss, Germany).

\section{Statistical Analysis}

The results are shown as a mean +/-SEM. All data were normalized to $2 \mathrm{DN}$ condition. All experiment was performed at least in 3 biological replicates. All statistical analyses were performed using GraphPad 
Prism 9.0 software (RRID:SCR_002798). Depending on the gaussian distribution we performed One-Way Analysis of variance (ANOVA) with Tukey post-hoc test or Kruskal-Wallis with post-hoc Dunn's test.

\section{Results}

\section{Optimization, characterization, and comparison of 3D model with 2D culture models.}

\section{Establishment of 3D cultures}

Irregular cell aggregates were observed especially for RCC model after using standard culture methods, e.g.: hanging drop in regular culture medium (Supplementary Fig. 1 A). Consistent spheroids from renal and melanoma murine cancer cells in their long-term culture in normoxic conditions were obtained by combining the hanging drop method with culture in semi-solid matrix (Fig.1B). 500 cells in a $20 \mu \mathrm{L}$ drop were seeded for both cell lines to compare the proliferative capacity of the cells during the sphere's formation. Spheroids were cultured for seven days in total: three days in hanging drops and then four days in agarose coated bottom plate in standard culture medium supplemented with methylcellulose (Fig.1A). After three days of culture in hanging drops irregular cell aggregates were observed for both cell lines: RenCa and B16F10 (Supplementary Fig.1 B). Round and regular spheroids were obtained after seven days of culture were completed (Fig1. B.). As measured by diameter, the RCC cells at the end of culture formed smaller spheroids $(\sim 400 \mu \mathrm{m})$ as compared to spheres $(\sim 600 \mu \mathrm{m})$ (Fig.1C) from B16F10 despite the initial number of seeded cells was the same. This suggests a slower growth of RenCa cells in 3D. Using flow cytometry, we determined the cell viability in 2D, and 3D cultures conducted in normoxic and hypoxic conditions. Higher proportion of viable cells for both cell lines was recovered from twodimensional cultures, than from spheroids in both oxygen tension conditions (Fig. 1 D). Microscopic observation of spheres stained by calcein and propidium iodide showed a necrotic core of 3D structures in normoxia (Fig.1.E). The cells from spheroids cultured in hypoxia showed a very low viability (Fig.1.D; Supplementary Fig. $1 \mathrm{C}$ ) and were smaller than spheres cultured in normoxia. It suggests that hypoxia which is known to develop in the center of the sphere may be influencing the viability of cells recovered from 3D structures where cells have an impaired access to oxygen (Klimkiewicz et al., 2017).

Consequently, spheroid culture in additional/external hypoxic conditions may cause an intense cellular stress drastically reducing the cell survival. As very few cells were recovered from hypoxia cultured spheres, we decided to conduct further research on cells cultured under normoxic condition which seems more appropriately reconstitute natural development of a tumor.

Fig. 1. Schematic model of spheroids formation (A), 500 cells/drop were seeded for both RenCa and B16F10 cells in standard medium with methylcellulose. After 3 days of culture cell aggregates were transferred into the agarose coated bottom plates and cultured for another 4 days. B- spheroids after 7 days of culture, $\mathbf{C}$ - comparison of spheroids diameter - $\mathrm{N}=3$. Effect of culture conditions on cells viability in different culture conditions measured by Flow Cytometry (D) and fluorescence staining of spheroids (E) by propidium iodide (orange - dead cells) and calcein (green - live cells), which shows the positions of 
live and dead cells in the spheres. Statistical analysis was performed by One-Way ANOVA/Tukey test *p<0,05, N=3 (2DN-control).

3D culture condition induces melanoma cancer cell cycle arrest at the GO/G1 phase

Spheroid culture affected the cell cycle distribution in both cell lines with no direct effect of hypoxia only (Fig 2 A-D). In the case of B16F10 cells, 3D culture caused accumulation of cells in G0/1 phase with concurrent reduction of S phase mostly (Fig. $2 \mathrm{~A}, \mathrm{C}$ ). On the contrary, spheroid recovered RenCa cells were enriched in proliferating, G2/M cells with non-significant drop of cells in both $\mathrm{G} 0 / 1$ and S phase (Fig.2 B, D). As activation of p53 tumor suppressor can lead to the cell cycle arrest (Schafer, 1998) we checked the expression of $p 53$ and its inhibitor $m d m 2$. For both cancer models we observed a similar downregulation of p53 expression by both hypoxia and 3D formation, as compared with 2D normoxic culture (Fig. $2 \mathrm{E}$ ). Expression of $m d m 2$ in melanoma cancer for 2D hypoxia and 3D was down regulated as compared with 2D normoxia, with a significantly stronger effect due to the spheroid type of culture (Fig. 2 E). For RCC cultures no statistically significant changes of $m d m 2$ expression were observed (Fig. $2 \mathrm{E}$ ).

\section{Fig. 2. Cell cycle analyzes by quantitative flow cytometry, in various culture conditions: $A, C-D N A$}

content frequency histograms of B16F10 and RenCa cells. B, D - quantitative measurements of cell cycle stages. E-Effect of culture conditions on the expression of proliferation capacity regulation genes. The expression of $m d m 2$ and $p 53$ for B16F10 and RenCa cell lines was determined by quantitative RT-PCR (qRT-PCR). $\beta$-actin served as a quantitative internal control. Statistical analysis was performed by OneWay ANOVA/Tukey test - * $\mathrm{p}<0,05, \mathrm{~N}=3$ (2DN-control).

3D melanoma cancer model induces hypoxia.

Presence of necrotic core in 3D structures of RCC and melanoma models after staining with calcein and propidium iodide suggested the induction of hypoxia in the middle of the spheres (Klimkiewicz et al., 2017). We checked several hypoxia related genes/proteins such as: HIF-1a, vegf-a, and VHL (Fig. 3. A, B). In 3D melanoma model we observed upregulation of hif-1a expression and a tendency for higher protein level as compared to both 2D normoxia and hypoxia (Fig. 3. A, B). Vh/ expression was not affected by any of tested culture conditions. Vegf expression increased both in 3D and 2D culture in hypoxia, although in the later condition, no HIF-1a upregulation occurred (Fig. 3. A, B). In RCC model we observed an opposite tendency; 3D culture downregulated hif $1 a, v h l$ and vegf-a genes, however hypoxia alone tended to increase HIF-1 a protein and $v h /$ and vegf gene expression (Fig. 3. A, B). Global gene expression analysis with NGS was performed for RCC model to identify hypoxia gene signatures in $3 D$ as compared with $2 D$ cultures (Fig. 3. C). String protein networks indicated upregulation of Endothelial PAS Domain Protein 1 (Epas1) for 3D culture as compared with both 2D normoxia and hypoxia culture conditions. Also, upregulation of matrix metalloproteinase-10 (Mmp10), Matrix metallopeptidase 13 (Mmp13) and Lysyl oxidase (Lox) was observed as compared to 2DN culture (Fig. 3. C).

Fig. 3. Effect of the culture conditions on the expression of hypoxia regulating genes $(A, B)$ and proteins (C). A-The gene expression of hif-1a, vhl and vegf-a was determined by quantitative RT-PCR (qRT-PCR); $\beta$ - 
actin served as a quantitative internal control. B - The protein expression of HIF-1a, VHL and VEGF assessed by Western blot; Vinculin served as a quantitative loading control. Statistical analysis was performed by One-Way ANOVA/Tukey test or Kruskal-Wallis/ Dunn's test - * $p<0,05, N \geq 3$ (2DN - control). C - Protein-protein interaction scaffold networks of EPAS1 determined by Next Generation Sequencing ( $q$ value $<0.05$ and $\left|\log _{2} \mathrm{FC}\right|>1,5$ ) identified in RenCa cells; Blue nodes represent proteins encoded by downregulated genes and red nodes represent proteins encoded by upregulated genes.

\section{Renal cancer spheroids show upregulation of a cancer stem like cell - CSC-population}

Upregulation of cells arrested in G0/G1 phase may suggest the presence of CSC (Caglar and Biray Avci, 2020), and hypoxia was shown to induce selection of CSC in cancer foci (Klimkiewicz et al., 2017). Therefore, the levels of several potential CSC markers such as: ALDH1, CD133 and CD105 were evaluated (Fig. 4). In the case of B16F10 cells with G0/G1 arrest, we could not observe CSCs induction; CD105 positive cells dropped in 3D culture while CD133 positive cells remained unchanged (Fig. 4 D, E). Similarly, ALDH1 was not significantly altered in those cells (Fig. 4 A, B). Surprisingly, in spheroid growing RCC cells, that were characterized by increased G2/M accumulation, a strong increase of ALDH1 protein level and activity were observed (Fig. 4 A, B). Analysis of String protein networks also indicated the upregulation of Aldehyde dehydrogenase 2 (ALDH2) expression in 3D (Fig. 4 C). Additionally, in RenCa cell line an increased number of CD133 positive cells in 2D cell cultures in hypoxia and in 3D cultures was shown, whereas no statistically significant changes for CD105 levels were observed (Fig 4. D, E). Remarkedly, an opposite tendency was displayed by B16F10 cells.

Fig. 4. Effect of the culture conditions on regulation of CSC. A - ALDH1 activity determined using flow cytometry method; B - protein expression of ALDH1 assessed by Western blot method; C - Protein-protein interaction scaffold networks of ALDH2 determined by Next Generation Sequencing ( $q$-value $<0.05$ and $\| \log _{2} \mathrm{FCl}>1,5$ ) identified in RenCa cells; Blue nodes represent proteins encoded by downregulated genes and red nodes represent proteins encoded by upregulated genes. $\mathbf{D}$ - evaluation of CD133 positive cells using flow cytometry method; $\mathbf{E}$ - evaluation of CD105 positive cells using flow cytometry method. Statistical analysis was performed by One-Way ANOVA/Tukey test or Kruskal-Wallis test/ Dunn's test ${ }^{*} \mathrm{p}<0,05, \mathrm{~N} \geq 3$.

\section{D induces EMT in melanoma model}

Another mechanism of cancer aggressiveness is EMT (Pastushenko and Blanpain, 2019). We tested whether this process is induced by spheroid culture by assessing the main EMT markers: Vimentin, $\mathrm{N}$ cadherin, and B-catenin (Fig. 5). In the B16 F10 melanoma model, the upregulation of N-cadherin, Vimentin and B-catenin was observed in spheroids. In the RenCa model B-catenin in spheroids was downregulated and $\mathrm{N}$-Cadherin could not be detected in any tested culture conditions (Fig. 5). Changes for vimentin expression in RCC models were not significant, however tendencies were opposite than those observed in melanoma model (Fig. 5), confirming the distinct reactions uncovered by the 3D mode of culturing the two cancer cell types. 
Fig. 5. Relative expression of EMT markers (A, B). A - relative protein expression of B-catenin, $\mathrm{N}$-cadherin and Vimentin assessed by Western blot; Vinculin served as a quantitative loading control, B- B-catenin, Ncadherin, and Vimentin detection by Western blots. Statistical analysis was performed by One-Way ANOVA/Tukey test, ${ }^{*} \mathrm{p}<0,05, \mathrm{~N} \geq 3$.

\section{D spheroid formation as an alternative model to in vivo murine tumors}

To assess for the significance of spheroid formation to mimic some tumor characteristics, we compared the levels of above tested markers in 3D models to in vivo tumors, comparatively to standard monolayers type of culture in normoxia (2DN).

3D cell culture models induce similar pattern of expression of cancer suppressor genes as in vivo growing tumor

Melanoma and RCC spheroids and their corresponding tumors, showed similar level of downregulation of p53 expression as compared to monolayer cultured cells (Fig. 6). While, in the melanoma model a similar downregulation pattern was observed for $m d m 2$ expression, this was not the case in the RCC model as $m d m 2$ expression was not significantly altered (although tended to increase) (Fig. 6).

Fig. 6. Comparison of proliferation capacity regulation genes. Relative expression of $m d m 2$ and $p 53$ for B16F10 and RenCa cells. All data normalized to 2DN expression. $\beta$-Actin served as a quantitative internal control. Statistical analysis was performed by One-Way ANOVA/Tukey test or Kruskal-Wallis test/ Dunn's test - * $p<0,05, N \geq 3$.

3D cultures and in vivo tumors show a similar expression pattern of proteins / genes associated with hypoxia

Melanoma spheroids and tumors displayed a similar upregulation of hif1a and vegf-a genes expression but the HIF-1 protein increase in spheroids was not as strong as in tumors (Fig. 7 A-C). A statistically significant downregulation of $\mathrm{vh} /$ was observed only for its transcripts in tumor (Fig. $7 \mathrm{~A}$ ). On the other hand, there was no effect on the corresponding protein level both in spheroid and tumor (Fig. 7 B, C). For RCC we observed that $3 \mathrm{D}$ and tumors show similar changes in expression when compared to monolayer, but tendencies were opposite to melanoma model: both in tumor and 3D a downregulation of hif1a, $\mathrm{vhl}$ and vegf-a was observed (Fig. $7 \mathrm{~A}-\mathrm{C}$ ). On the level of proteins, VHL increased in tumors only; in spheroid culture this upregulation did not reach a statistical significance (Fig. 7 B, C).

Fig.7. Comparison of the expression of hypoxia regulating genes $(A, D)$ and proteins $(B, C, E, F)$. A, D - The gene expression of hif1a, vhl and vegf-a was determined by quantitative RT-PCR (qRT-PCR); $\beta$-actin served as a quantitative internal control. B, E - The protein expression of HIF1a, VHL and VEGF assessed by Western blot; Vinculin served as a quantitative loading control C, F - HIF1a and VHL detection by Western blots. Statistical analysis was performed by One-Way ANOVA/Tukey test or Kruskal-Wallis/ Dunn's test ${ }^{*} \mathrm{p}<0,05, \mathrm{~N} \geq 3$ (2DN - control). 
RCC spheroids and tumors show a similar expression pattern of markers associated with stemness

Previously observed ALDH1 induction in renal cancer spheroids was also seen tumors; ALDH1 protein level was increased similarly in 3D culture as compared to monolayer (Fig. 8). Also, melanoma tumors were characterized by a very high expression of this protein, although in this model, spheroid culture could not upregulate ALDH1 significantly (Fig. 8).

Fig 8. Comparisons of CSC expression in 3D and Tumor (A, B) A-Relative to $2 \mathrm{DN}$ protein expression of ALDH1, Vinculin served as a quantitative loading control. B- ALDH1 detection by Western blots. Statistical analysis was performed by One-Way ANOVA/Tukey test $p<0,05, \mathrm{~N} \geq 3$ (2DN - control).

Melanoma and RCC spheroids and tumors show a similar expression pattern of markers associated with EMT

Previously observed ß-catenin induction in melanoma and downregulation in renal cancer spheroids was also observed tumors (Fig. 8). The same tendencies for vimentin expression in 3D and the tumor was also observed for both models, were we observed upregulation of this protein in melanoma and downregulation in RCC model (Fig. 8).

Fig 9. Comparisons of EMT markers expression in 3D and Tumor (A, B) A-Relative to 2DN protein expression of $ß$-catenin and Vimentin, Vinculin served as a quantitative loading control. B- Vimentin and ß-catenin detection by Western blots. Statistical analysis was performed by One-Way ANOVA/Tukey test or Kruskal-Wallis/ Dunn's test $\mathrm{p}<0,05, \mathrm{~N} \geq 3$ (2DN - control).

\section{Discussion}

To increase validity and success rate of cancer research, it is necessary to handle parameters that mimic the most important features of the disease. Monolayer cell cultures provide useful and homogenous model, however many studies showed that 3D culture models display features that are closer to in vivo tumor characteristics (Klimkiewicz et al., 2017; Ravi et al., 2015). 3D models using B16F10 cells do exist for murine melanoma, but there are no well-optimized models for murine kidney cancer using RenCa cells for studies (Goundiam et al., 2010; Debnath et al., 2021; Ulukan et al., 2020; Klimkiewicz et al., 2017). The present work performed long long-term 3D cultures allowing comparative models for melanoma and renal cancer and characterized them in terms of response to hypoxia, CSC selection and EMT induction. Importantly, we demonstrated the validity of the spheroid type of culture to reflect the mechanisms observed in tumors in vivo. The designed method does not require any special equipment nor expensive laboratory consumables and pipetting was reduced to minimum. We exploited 96-well format that can be easily used for cost- and time-effective drug screening but provide sufficient cellular material for molecular experiments (western blot, qPCR or flow cytometry can be performed using 96 spheroids for each technique) (Fig. 1 A). 
It was shown that size of the sphere influences the cellular processes induced within. The dimensions of spheroids are usually correlated with seeding density (Singh et al., 2020), however in our study we point out also intrinsic features of used cells for the outcome of 3D culture. The study, although restricted to two murine cancer cell type to reduce the variability of spheroid formation, confirms the universality of the method. Indeed, the present modification of hanging drop method was efficiently inducing 3D formation by both cell lines, despite the poor RenCa ability for sphere formation (Supplementary Fig. 1 A, B) (Fig.1C). RCC spheroids were significantly smaller than melanomas, although initial cell number was same for both cell lines. Such differences may result from different proliferation capacity of cells, resulting is a less apparent necrotic core (Fig $1 \mathrm{E}$ ). Ability to form spheroids corelates with cancer stemlike cells characteristics of cell lines (Y. Zhang et al., 2013; Debeb et al., 2010). We showed that melanoma cell line is initially characterized by higher levels of ALDH1 (protein / ALDH1 activity) and CD133 positive cells than RenCa cells (Fig. 4) what relates with the higher capacity of B16F10 cells to form spheroids.

Cells recovered from spheroids were less viable probably due to a necrotic core formed in 3D structures as observed by microscopy (Fig. $1 \mathrm{E}$ ). This suggests that hypoxia develops in the center of the sphere (Riffle and Hegde, 2017). Indeed, in the melanoma spheres, we observed upregulation of Hif1 a (Semenza, 2001), inducer of the main proangiogenic factor VEGF-A, (Fig. 3 A). In RCC 3D model we observed downregulation hif $1 a, v h /$ and vegf-a expression (not statistically significant), which suggest that hypoxic processes were not effectively induced probably because spheres were smaller (Fig. 3 A, B). However, the same pattern of gene expression was observed in the subcutaneous tumor obtained in vivo (Fig 7). This shows that 3D culture induces cellular mechanisms that are cell-specific, in addition to the physical parameters that are universal and due to spheroid architecture. This supports the conclusion that, in many aspects, spheroids are sufficient to model tumor physiology and present a valuable alternative model to in vivo experiments.

Further characterization of RenCa spheroid model revealed upregulation of Epas1 (Hif2a) transcript (Fig. $3 \mathrm{C}$ ). Studies using in vivo and in vitro models indicated that HIF-1a acts as a tumor suppressor, whereas HIF-2a has oncogenic potential (Schödel et al., 2016). Together with increased expression of Hif2a, increase of Mmp10, Mmp13 and Lox expression was observed (Fig. $3 \mathrm{C}$ ). These proteins are associated with cancer progression and metastasis in various types of tumors (Leo et al., 2018; Yamada et al., 2010; G. Zhang et al., 2014). Taken together that RCC spheroids have population of aggressive, highly proliferative cells that are selected upon 3D formation. Indeed, RenCa spheroids were enriched in proliferating, G2/M cells (Fig. 2), cancer stem-like cells (upregulation of ALDH1, ALDH2 expression and CD133+ increased population) (Fig. 4) and metastasis linked proteins. While the induction of Hif2a down regulator for $p 53$ (Cho et al., 2016; Bertout et al., 2009) was observed in both models (Fig $2 \mathrm{E}$ ), induction of pro-metastatic EMT-linked processes was not observed (Pastushenko and Blanpain, 2019) in RCC spheres (Fig 5).

In contrast melanoma spheroids were characterized by a reduced percentage of proliferating cells (G2/M) with a simultaneous $\mathrm{G0} / \mathrm{G} 1$ phase shift in the cell cycle, despite $p 53$ downregulation together with its 
regulator $m d m 2$ (Fig. 2). G0/G1 phase arrest also suggests the presence of quiescent cancer stem cells (Das et al., 2020), although other markers of CSC phenotype, as ALDH1 and CD133, were not induced in B16F10 spheroids (Fig. 4 A, B, D) or down regulated as CD105 positive cells (Saeednejad Zanjani et al., 2018) (Fig. 4 E). CD105 downregulation in these cells causes reduction of proliferative capacity, and promotes spheroids compaction (Dolinsek et al., 2015). This may explain cell cycle arrest in G0/G1 phase of cells in the melanoma spheres (Fig. 2 A-D), and shape differences of both cell models (Fig. $1 \mathrm{~B}, \mathrm{E}$ ). Although we do not observe any changes in CSC markers expression in melanoma spheres, EMT markers were upregulated (Fig. 5). The use of the 3D type of culture uncovered the distinct molecular mechanisms set by the melanoma and the RCC cells to reach a higher aggressiveness. As EMT is induced by hypoxia in several types of cancer (Joseph et al., 2018), this might have not been reached by RenCa spheres in contrast to B16F10. Although RenCa spheroids are smaller and less hypoxic, their structure allowed a positive CSC selection and proliferation. B16F10 form bigger and hypoxic spheres which possess EMT features. Our result correspond with what was shown previously, that melanoma intra-tumor heterogeneity does not rely on the CSC hypothesis, but rather on EMT features (Goding and Arnheiter, 2019).

To validate the developed models for mimicking the most important features of cancer, we compared the levels of the above tested markers in 3D models with in vivo tumors. Melanoma and RCC spheroids showed a similar pattern of regulation of $p 53$ oncogene and its regulator - mdm2 (Fig. 6), which may indicate similar proliferative capacities of tumors and spheres (Tazawa et al., 2016). Hypoxia markers found in the melanoma spheroids were expressed at similar levels but the HIF1 protein, for which increase in spheroid lower than in the tumor (Fig. 7). This indicates that melanoma 3D culture is indeed able to mimic the features of tumor hypoxia. RCC spheres also show similar changes as observed in the corresponding tumor, although these two cancer types were often oppositely modulating molecular pathways (Fig. 7). The 3D type of cultures clearly brings conditions that are much closer to in vivo ones. Indeed, the induction of CSC in RCC model was as efficient as in the tumor confirming the similarities between 3D and the tumor, in contrast to the melanoma where the CSC induction was lower than in the tumor but in both cases the EMT processes were largely mimicked.

\section{Conclusion}

In our studies, we observed that 3D models can mimic some features of the tumor such as hypoxia and CSC induction, adequately to the characteristics of corresponding in vivo tumor mass. Spheroid models of renal and melanoma cancers induce different mechanisms, related to the progression of cancer. RCC model displayed induction of CSC characteristic whereas, melanoma spheres contribute to EMT-related mechanism. Changes in cellular processes in these two cell lines in spheroids compared to standard monolayer were not universal, however were reproduced in the in vivo subcutaneous model, proving the validity of spheroid cultures to model cancer physiology as an alternative to animal experiments, at least in initial steps of research. 


\section{References}

Abou Khouzam, R., Brodaczewska, K., Filipiak, A., Zeinelabdin, N. A., Buart, S., Szczylik, C., et al. (2020). Tumor Hypoxia Regulates Immune Escape/Invasion: Influence on Angiogenesis and Potential Impact of Hypoxic Biomarkers on Cancer Therapies. Front Immunol, 11, 613114. doi:10.3389/fimmu.2020.613114.

Abourbih, S., Sircar, K., Tanguay, S., Kassouf, W., Aprikian, A., Mansure, J., et al. (2013). Aldehyde dehydrogenase 1 expression in primary and metastatic renal cell carcinoma: an immunohistochemistry study. World J Surg Oncol, 11, 298. doi:10.1186/1477-7819-11-298.

Bertout, J. A., Majmundar, A. J., Gordan, J. D., Lam, J. C., Ditsworth, D., Keith, B., et al. (2009). HIF2alpha inhibition promotes p53 pathway activity, tumor cell death, and radiation responses. Proc Natl Acad Sci U $S A, 106(34), 14391-6$. doi:10.1073/pnas.0907357106.

Bradley, J. R., Wang, J., Pacey, S., Warren, A. Y., Pober, J. S., \& Al-Lamki, R. S. (2020). Tumor necrosis factor receptor-2 signaling pathways promote survival of cancer stem-like CD133. FASEB Bioadv, 2(2), 126-144. doi:10.1096/fba.2019-00071.

Bray, F., Ferlay, J., Soerjomataram, I., Siegel, R. L., Torre, L. A., \& Jemal, A. (2018). Global cancer statistics 2018: GLOBOCAN estimates of incidence and mortality worldwide for 36 cancers in 185 countries. $C A$ Cancer J Clin, 68(6), 394-424. doi:10.3322/caac.21492.

Caglar, H. O., \& Biray Avci, C. (2020). Alterations of cell cycle genes in cancer: unmasking the role of cancer stem cells. Mol Biol Rep, 47(4), 3065-3076. doi:10.1007/s11033-020-05341-6.

Carreau, A., El Hafny-Rahbi, B., Matejuk, A., Grillon, C., \& Kieda, C. (2011). Why is the partial oxygen pressure of human tissues a crucial parameter? Small molecules and hypoxia. J Cell Mol Med, 15(6), 1239-53. doi:10.1111/j.1582-4934.2011.01258.x.

Casanovas, O., Hicklin, D. J., Bergers, G., \& Hanahan, D. (2005). Drug resistance by evasion of antiangiogenic targeting of VEGF signaling in late-stage pancreatic islet tumors. Cancer Cell, 8(4), 299309. doi:10.1016/j.ccr.2005.09.005.

Cheon, D. J., \& Orsulic, S. (2011). Mouse models of cancer. Annu Rev Pathol, 6, 95-119. doi:10.1146/annurev.pathol.3.121806.154244.

Cho, H., Du, X., Rizzi, J. P., Liberzon, E., Chakraborty, A. A., Gao, W., et al. (2016). On-target efficacy of a HIF2a antagonist in preclinical kidney cancer models. Nature, 539(7627), 107-111. doi:10.1038/nature19795.

Collet, G., Skrzypek, K., Grillon, C., Matejuk, A., El Hafni-Rahbi, B., Lamerant - Fayel, N., et al. (2012). Hypoxia control to normalize pathologic angiogenesis: Potential role for endothelial precursor cells and miRNAs regulation. Vascular Pharmacology, 56(5-6), 252-261. doi:10.1016/j.vph.2012.03.001. 
Cowey, C. L., \& Rathmell, W. K. (2009). VHL gene mutations in renal cell carcinoma: role as a biomarker of disease outcome and drug efficacy. Curr Oncol Rep, 11(2), 94-101. doi:10.1007/s11912-009-0015-5.

Das, P. K., Islam, F., \& Lam, A. K. (2020). The Roles of Cancer Stem Cells and Therapy Resistance in Colorectal Carcinoma. Cells, 9(6). doi:10.3390/cells9061392.

Debeb, B. G., Zhang, X., Krishnamurthy, S., Gao, H., Cohen, E., Li, L., et al. (2010). Characterizing cancer cells with cancer stem cell-like features in 293T human embryonic kidney cells. Mol Cancer, 9, 180. doi:10.1186/1476-4598-9-180.

Debnath, S., Mukherjee, A., Saha, D., Dash, J., \& Chatterjee, T. K. (2021). Poly-I-Lysine inhibits VEGF and cMyc mediated tumor-angiogenesis and induces apoptosis in 2D and 3D tumor microenvironment of both MDA-MB-231 and B16F10 induced mice model. Int J Biol Macromol, 183, 528-548.

doi:10.1016/j.jijbiomac.2021.04.109.

Dolinsek, T., Sersa, G., Prosen, L., Bosnjak, M., Stimac, M., Razborsek, U., et al. (2015). Electrotransfer of Plasmid DNA Encoding an Anti-Mouse Endoglin (CD105) shRNA to B16 Melanoma Tumors with Low and High Metastatic Potential Results in Pronounced Anti-Tumor Effects. Cancers (Basel), 8(1). doi:10.3390/cancers8010003.

Duval, K., Grover, H., Han, L. H., Mou, Y., Pegoraro, A. F., Fredberg, J., et al. (2017). Modeling Physiological Events in 2D vs. 3D Cell Culture. Physiology (Bethesda), 32(4), 266-277. doi:10.1152/physiol.00036.2016.

Goding, C. R., \& Arnheiter, H. (2019). MITF-the first 25 years. Genes Dev, 33(15-16), 983-1007. doi:10.1101/gad.324657.119.

Goundiam, O., Nagel, M. D., \& Vayssade, M. (2010). Growth and survival signalling in B16F10 melanoma cells in 3D culture. Cell Biol Int, 34(4), 385-91. doi:10.1042/CBI20090147.

Hinshaw, D. C., \& Shevde, L. A. (2019). The Tumor Microenvironment Innately Modulates Cancer Progression. Cancer Res, 79(18), 4557-4566. doi:10.1158/0008-5472.CAN-18-3962.

Hoarau-Véchot, J., Rafii, A., Touboul, C., \& Pasquier, J. (2018). Halfway between 2D and Animal Models: Are 3D Cultures the Ideal Tool to Study Cancer-Microenvironment Interactions? Int J Mol Sci, 19(1). doi:10.3390/ijms19010181.

Jeon, S., Lee, H. S., Lee, G. Y., Park, G., Kim, T. M., Shin, J., et al. (2017). Shift of EMT gradient in 3D spheroid MSCs for activation of mesenchymal niche function. Sci Rep, 7(1), 6859. doi:10.1038/s41598017-07049-3.

Joseph, J. P., Harishankar, M. K., Pillai, A. A., \& Devi, A. (2018). Hypoxia induced EMT: A review on the mechanism of tumor progression and metastasis in OSCC. Oral Oncol, 80, 23-32. doi:10.1016/j.oraloncology.2018.03.004. 
Khacho, M., Mekhail, K., Pilon-Larose, K., Payette, J., \& Lee, S. (2008). Cancer-causing mutations in a novel transcription-dependent nuclear export motif of $\mathrm{VHL}$ abrogate oxygen-dependent degradation of hypoxiainducible factor. Mol Cell Biol, 28(1), 302-14. doi:10.1128/MCB.01044-07.

Klimkiewicz, K., Weglarczyk, K., Collet, G., Paprocka, M., Guichard, A., Sarna, M., et al. (2017). A 3D model of tumour angiogenic microenvironment to monitor hypoxia effects on cell interactions and cancer stem cell selection. Cancer Lett, 396, 10-20. doi:10.1016/j.canlet.2017.03.006.

Leo, C., Cotic, C., Pomp, V., Fink, D., \& Varga, Z. (2018). Overexpression of Lox in triple-negative breast cancer. Ann Diagn Pathol, 34, 98-102. doi:10.1016/j.anndiagpath.2018.03.009.

Li, C., Lu, J., \& Zhang, B. (2012). Development of a novel chronic intermittent hypoxia chamber. Sleep Breath, 16(1), 177-9. doi:10.1007/s11325-010-0470-0.

Melissaridou, S., Wiechec, E., Magan, M., Jain, M. V., Chung, M. K., Farnebo, L., et al. (2019). The effect of 2D and 3D cell cultures on treatment response, EMT profile and stem cell features in head and neck cancer. Cancer Cell Int, 19, 16. doi:10.1186/s12935-019-0733-1.

Pan, Y., Ma, S., Cao, K., Zhou, S., Zhao, A., Li, M., et al. (2018). Therapeutic approaches targeting cancer stem cells. J Cancer Res Ther, 14(7), 1469-1475. doi:10.4103/jcrt.JCRT_976_17.

Pastushenko, I., \& Blanpain, C. (2019). EMT Transition States during Tumor Progression and Metastasis. Trends Cell Biol, 29(3), 212-226. doi:10.1016/j.tcb.2018.12.001.

Quail, D. F., \& Joyce, J. A. (2013). Microenvironmental regulation of tumor progression and metastasis. Nat Med, 19(11), 1423-37. doi:10.1038/nm.3394.

Rathmell, W. K., \& Chen, S. (2008). VHL inactivation in renal cell carcinoma: implications for diagnosis, prognosis and treatment. Expert Rev Anticancer Ther, 8(1), 63-73. doi:10.1586/14737140.8.1.63.

Ravi, M., Paramesh, V., Kaviya, S. R., Anuradha, E., \& Solomon, F. D. (2015). 3D cell culture systems: advantages and applications. J Cell Physiol, 230(1), 16-26. doi:10.1002/jcp.24683.

Riffle, S., \& Hegde, R. S. (2017). Modeling tumor cell adaptations to hypoxia in multicellular tumor spheroids. J Exp Clin Cancer Res, 36(1), 102. doi:10.1186/s13046-017-0570-9.

Saeednejad Zanjani, L., Madjd, Z., Abolhasani, M., Shariftabrizi, A., Rasti, A., \& Asgari, M. (2018). Expression of CD105 cancer stem cell marker in three subtypes of renal cell carcinoma. Cancer Biomark, 21(4), 821-837. doi:10.3233/CBM-170755.

Sakashita, M., Tanaka, T., \& Nangaku, M. (2019). Hypoxia-Inducible Factor-Prolyl Hydroxylase Domain Inhibitors to Treat Anemia in Chronic Kidney Disease. Contributions to Nephrology, 198, 112-123. doi:10.1159/000496531. 
Schafer, K. A. (1998). The cell cycle: a review. Vet Pathol, 35(6), 461-78. doi:10.1177/030098589803500601.

Schödel, J., Grampp, S., Maher, E. R., Moch, H., Ratcliffe, P. J., Russo, P., et al. (2016). Hypoxia, Hypoxiainducible Transcription Factors, and Renal Cancer. Eur Urol, 69(4), 646-657. doi:10.1016/j.eururo.2015.08.007.

Semenza, G. L. (2001). HIF-1 and mechanisms of hypoxia sensing. Curr Opin Cell Biol, 13(2), 167-71.

Shi, D., Che, J., Yan, Y., Peng, B., Yao, X., \& Guo, C. (2019). Expression and clinical value of CD105 in renal cell carcinoma based on data mining in The Cancer Genome Atlas. Exp Ther Med, 17(6), 4499-4505. doi:10.3892/etm.2019.7493.

Simbulan-Rosenthal, C. M., Dougherty, R., Vakili, S., Ferraro, A. M., Kuo, L. W., Alobaidi, R., et al. (2019). CRISPR-Cas9 Knockdown and Induced Expression of CD133 Reveal Essential Roles in Melanoma Invasion and Metastasis. Cancers (Basel), 11(10). doi:10.3390/cancers11101490.

Singh, S. K., Abbas, S., Saxena, A. K., Tiwari, S., Sharma, L. K., \& Tiwari, M. (2020). Critical role of threedimensional tumorsphere size on experimental outcome. Biotechniques, 69(5), 333-338. doi:10.2144/btn2020-0081.

Taylor, L. A., Abraham, R. M., Tahirovic, E., van Belle, P., Li, B., Huang, L., et al. (2017). High ALDH1 expression correlates with better prognosis in tumorigenic malignant melanoma. Mod Pathol, 30(5), 634639. doi:10.1038/modpathol.2016.226.

Tazawa, H., Kagawa, S., \& Fujiwara, T. (2016). p53 Replacement Therapy for Cancer. Recent Results Cancer Res, 209, 1-15. doi:10.1007/978-3-319-42934-2_1.

Ulukan, B., Bihorac, A., Sipahioglu, T., Kiraly, R., Fesus, L., \& Telci, D. (2020). Role of Tissue Transglutaminase Catalytic and Guanosine Triphosphate-Binding Domains in Renal Cell Carcinoma Progression. ACS Omega, 5(43), 28273-28284. doi:10.1021/acsomega.0c04226.

Verjans, E. T., Doijen, J., Luyten, W., Landuyt, B., \& Schoofs, L. (2018). Three-dimensional cell culture models for anticancer drug screening: Worth the effort? J Cell Physiol, 233(4), 2993-3003. doi:10.1002/jcp.26052.

Vlashi, E., \& Pajonk, F. (2015). Cancer stem cells, cancer cell plasticity and radiation therapy. Semin Cancer Biol, 31, 28-35. doi:10.1016/j.semcancer.2014.07.001.

Yamada, T., Oshima, T., Yoshihara, K., Tamura, S., Kanazawa, A., Inagaki, D., et al. (2010). Overexpression of MMP-13 gene in colorectal cancer with liver metastasis. Anticancer Res, 30(7), 2693-9.

Zhang, G., Miyake, M., Lawton, A., Goodison, S., \& Rosser, C. J. (2014). Matrix metalloproteinase-10 promotes tumor progression through regulation of angiogenic and apoptotic pathways in cervical 
tumors. BMC Cancer, 14, 310. doi:10.1186/1471-2407-14-310.

Zhang, J., \& Zhang, Q. (2018). VHL and Hypoxia Signaling: Beyond HIF in Cancer. Biomedicines, 6(1). doi:10.3390/biomedicines6010035.

Zhang, Y., Sun, B., Zhao, X., Liu, Z., Wang, X., Yao, X., et al. (2013). Clinical significances and prognostic value of cancer stem-like cells markers and vasculogenic mimicry in renal cell carcinoma. J Surg Oncol, 108(6), 414-9. doi:10.1002/jso.23402.

\section{Abbreviations}

2D: two-dimensional culture

2DH: two-dimensional culture in hypoxia

2DN: two-dimensional culture in normoxia

3D: three-dimensional culture

ALDH1: Aldehyde dehydrogenase 1

ALDH1: Aldehyde dehydrogenase 2

ANOVA: Analysis of variance

CSC: Cancer Stem Cells

EMT: Epithelial Mesenchymal Transition

EPAS1: Endothelial PAS Domain Protein 1

HIF1a: Hypoxia Inducible Factor 1a

Lox: Lysyl oxidase

Mmp10: matrix metalloproteinase-10

Mmp13: Matrix metallopeptidase 13

NGS: Next Generation Sequencing

p02: partial pressure of oxygen

RCC: renal cancer

VEGF-A: Vascular endothelial growth factor A 
VHL: the tumor suppressor von Hippel-Lindau

\section{Declarations}

Ethics approval and consent to participate:

All experiments were conducted after obtaining approval for procedures from Second Warsaw Local Ethics Committee for Animal Experimentation (no. WAW2/76/2017) and following Directive 2010/63/EU regulations.

Consent for publication: Not applicable

Availability of data and materials:

The datasets generated and/or analyzed during the current study are available in the [Mendeley Data] repository, [doi:10.17632/9259s6c46f.1].

Competing interests:

The authors declare that they have no competing interests.

Funding: The research was funded by National Scientific Center grant no 2016/23/B/NZ1/03211. 3D culture optimalization was performed within Military Institute of Medicine intramural grant no 5/8958 (467). AF was supported by European Social Fund: POWER "Next generation sequencing technologies in biomedicine and personalized medicine"

Autor contributions: AF, KB, AM performed experiments and analyzed the data. CK developed conception of the research and work hypothesis. All authors contributed to the writing of this manuscript

Acknowledgements: Not applicable.

\section{Figures}




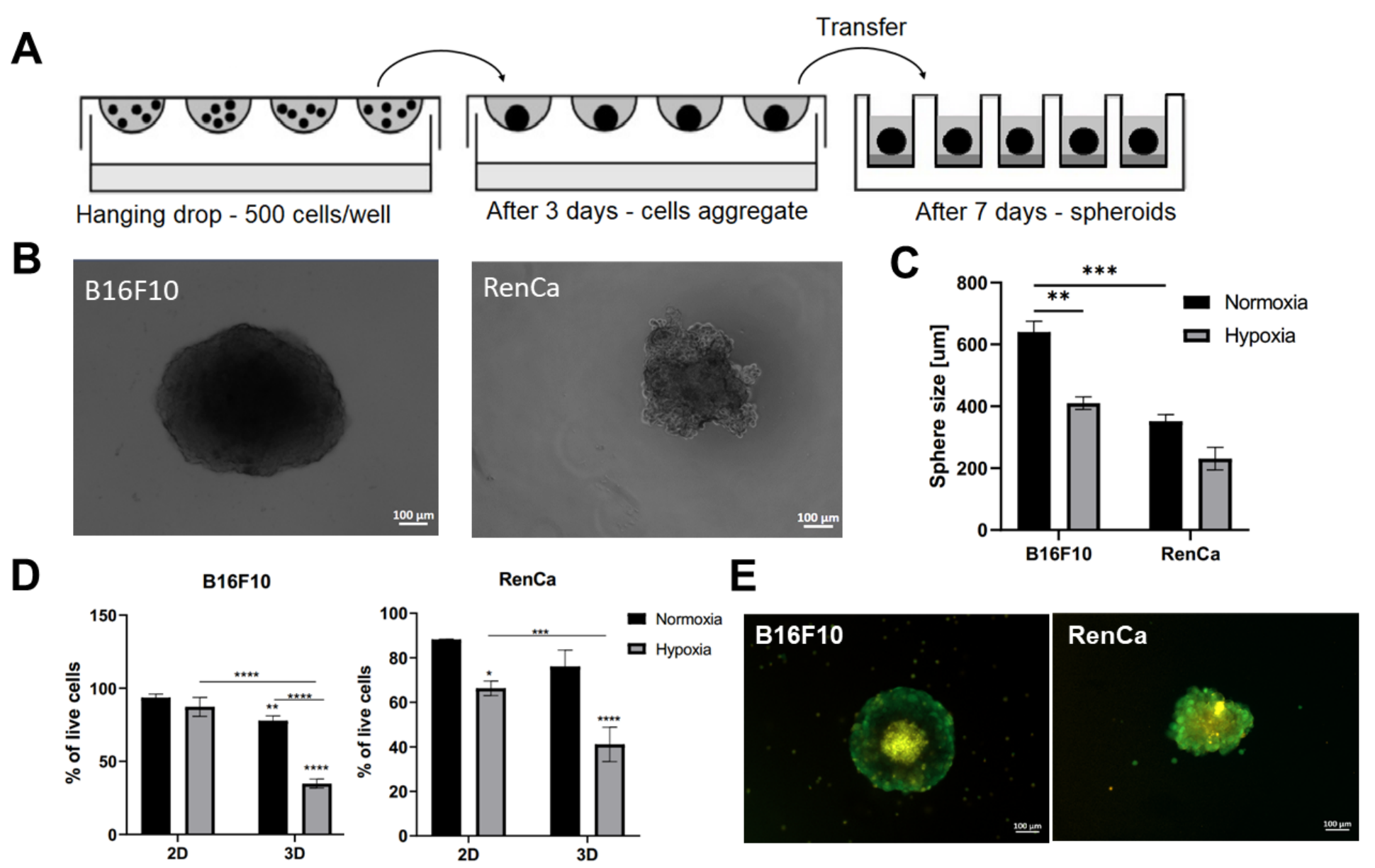

\section{Figure 1}

500 cells/drop were seeded for both RenCa and B16F10 cells in standard medium with methylcellulose. After 3 days of culture cell aggregates were transferred into the agarose coated bottom plates and cultured for another 4 days. B- spheroids after 7 days of culture, $\mathbf{C}$ - comparison of spheroids diameter $\mathrm{N}=3$. Effect of culture conditions on cells viability in different culture conditions measured by Flow Cytometry (D) and fluorescence staining of spheroids (E) by propidium iodide (orange - dead cells) and calcein (green - live cells), which shows the positions of live and dead cells in the spheres. Statistical analysis was performed by One-Way ANOVA/Tukey test - * $\mathrm{p}<0,05, \mathrm{~N}=3$ (2DN-control). 

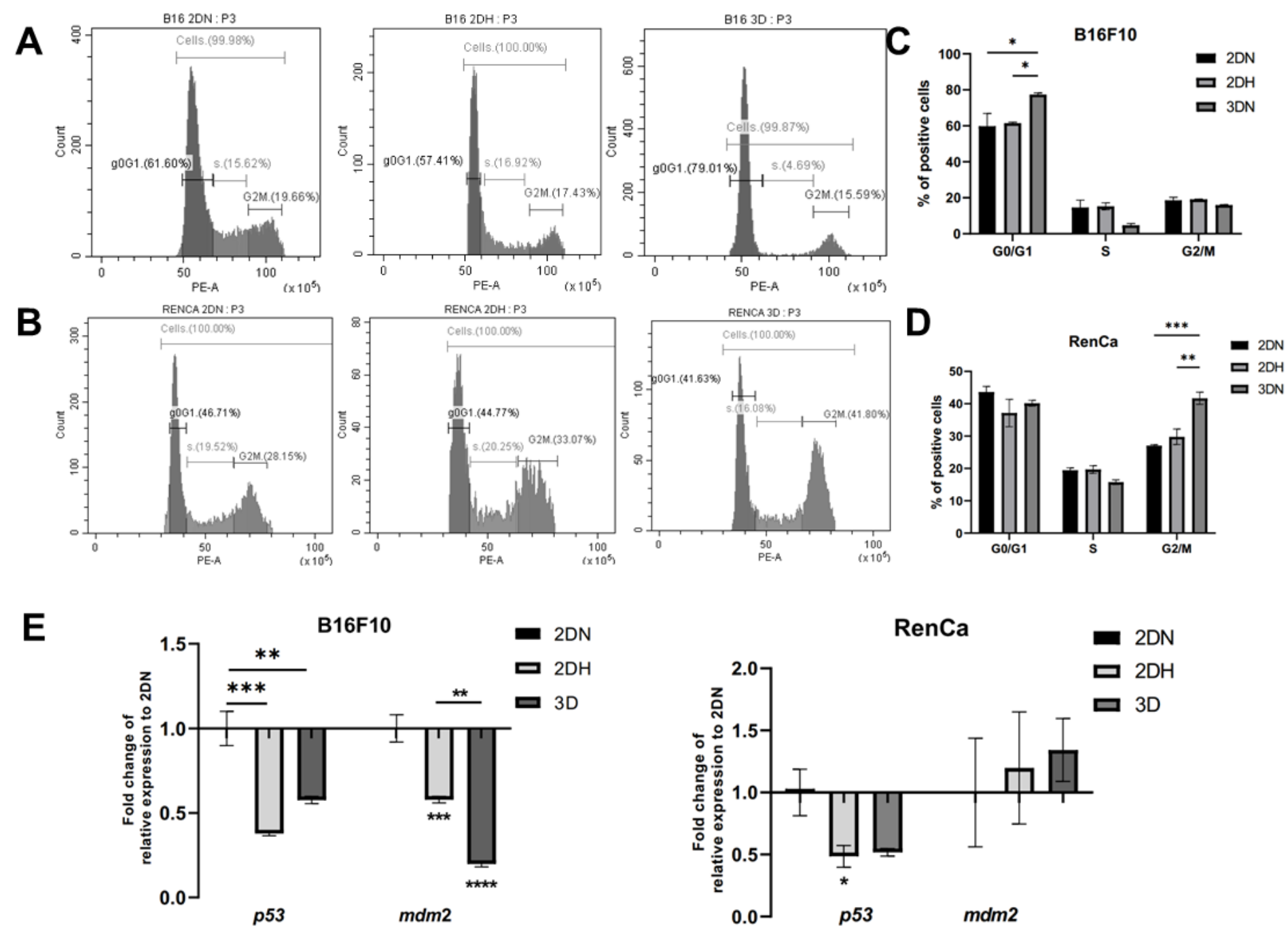

Figure 2

Cell cycle analyzes by quantitative flow cytometry, in various culture conditions: A, C - DNA content frequency histograms of B16F10 and RenCa cells. B, D - quantitative measurements of cell cycle stages. $\mathrm{E}$ - Effect of culture conditions on the expression of proliferation capacity regulation genes. The expression of $m d m 2$ and $p 53$ for $\mathrm{B} 16 \mathrm{~F} 10$ and RenCa cell lines was determined by quantitative RT-PCR (qRT-PCR). $\beta$-actin served as a quantitative internal control. Statistical analysis was performed by OneWay ANOVA/Tukey test - * $\mathrm{p}<0,05, \mathrm{~N}=3$ (2DN-control). 
A
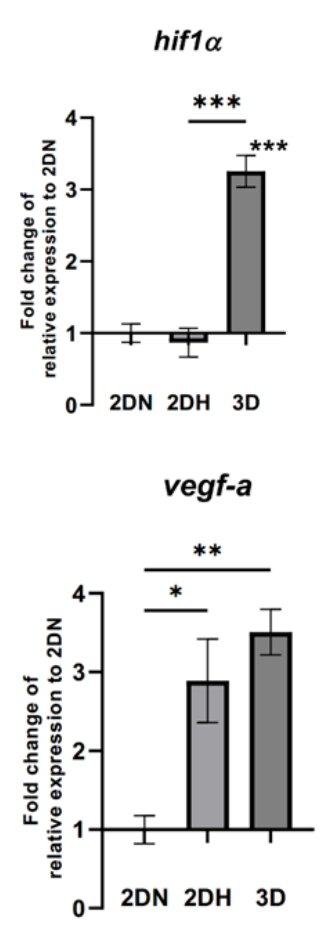

$v h l$

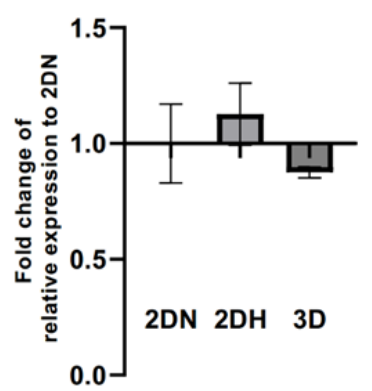

RenCa

hif1 $\alpha$

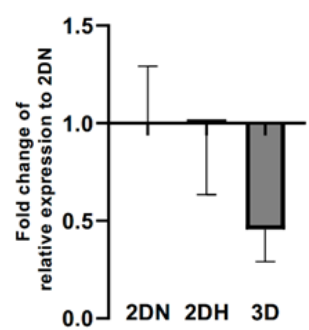

vegf-a

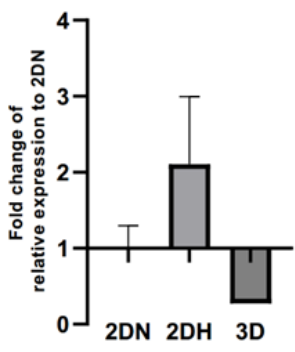

vhl
B

B16F10

VHL
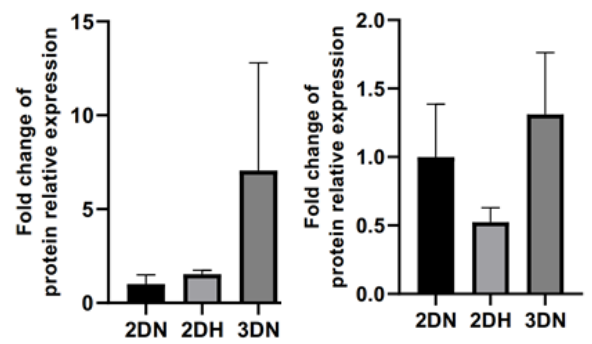

RenCa

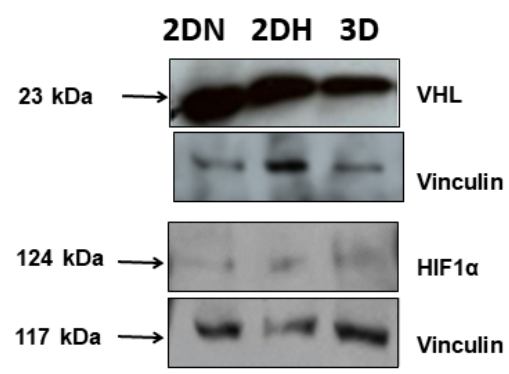

VHL

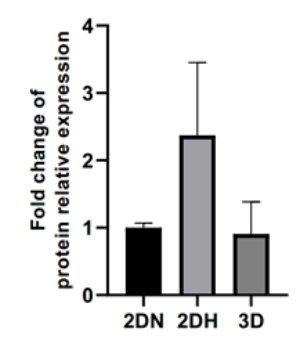

3D vs 2DN

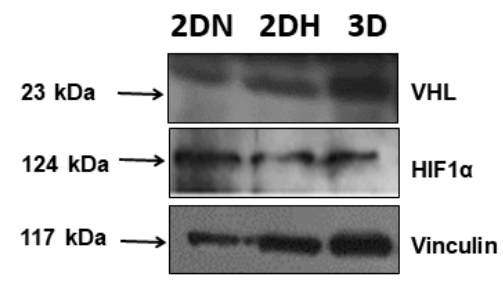

C

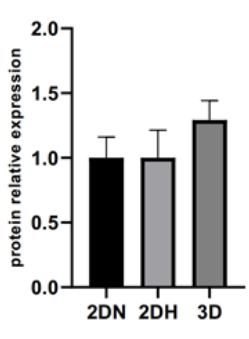

3D vs $2 \mathrm{DH}$
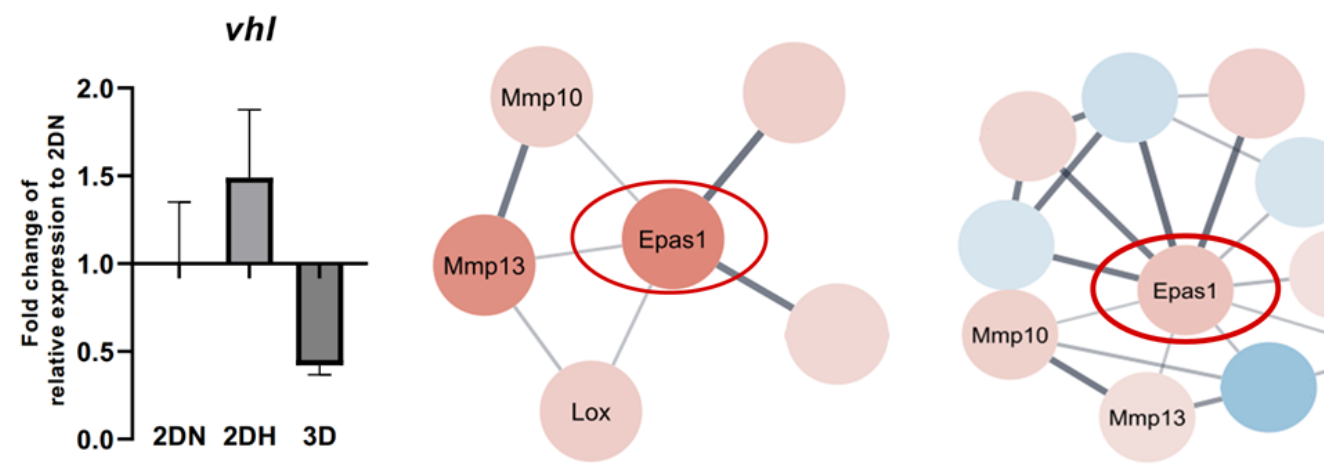

Figure 3

Effect of the culture conditions on the expression of hypoxia regulating genes (A, B) and proteins (C). A The gene expression of hif- $1 a, v h /$ and vegf-a was determined by quantitative RT-PCR (qRT-PCR); $\beta$-actin served as a quantitative internal control. B - The protein expression of HIF-1a, VHL and VEGF assessed by Western blot; Vinculin served as a quantitative loading control. Statistical analysis was performed by One-Way ANOVA/Tukey test or Kruskal-Wallis/ Dunn's test - * $p<0,05, \mathrm{~N} \geq 3$ (2DN - control). C - Proteinprotein interaction scaffold networks of EPAS1 determined by Next Generation Sequencing ( $q$-value < 0.05 and $\left.\left|\log _{2} \mathrm{FC}\right|>1,5\right)$ identified in RenCa cells; Blue nodes represent proteins encoded by downregulated genes and red nodes represent proteins encoded by upregulated genes. 

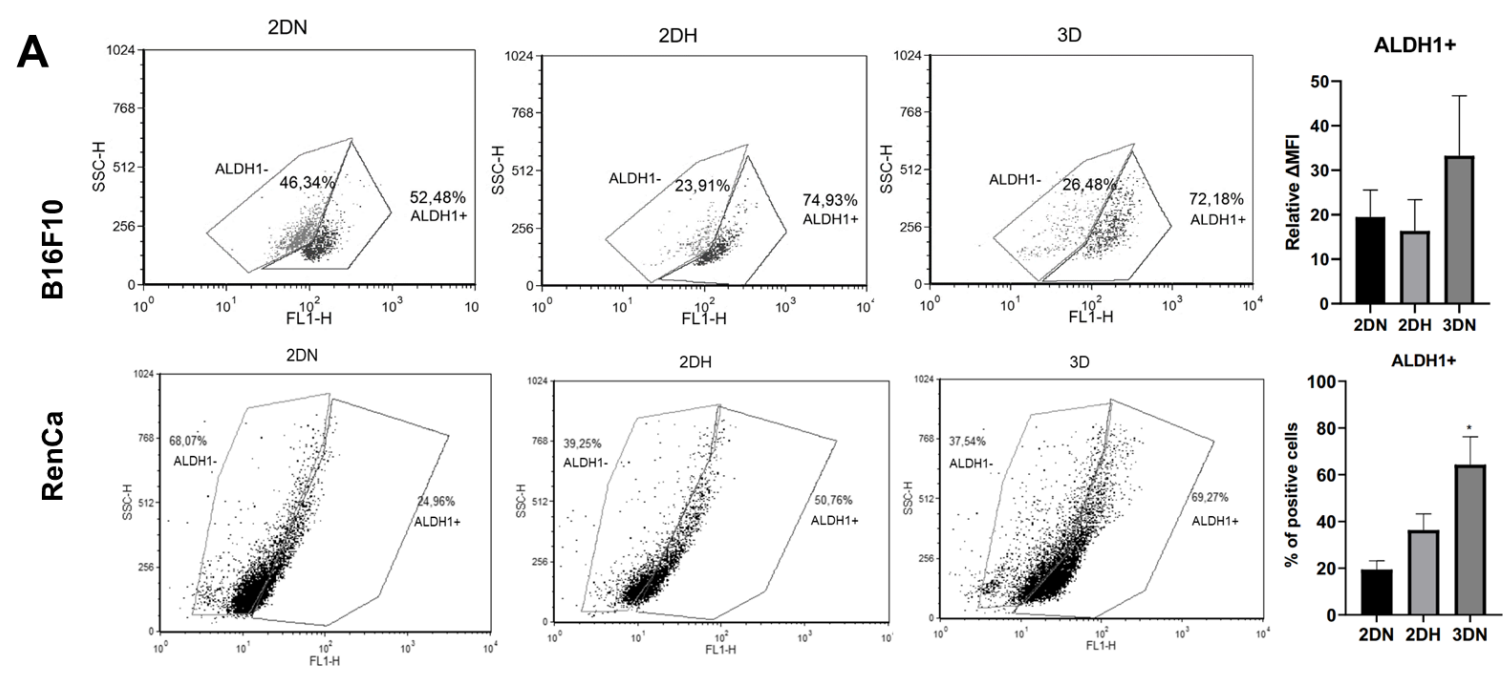

B

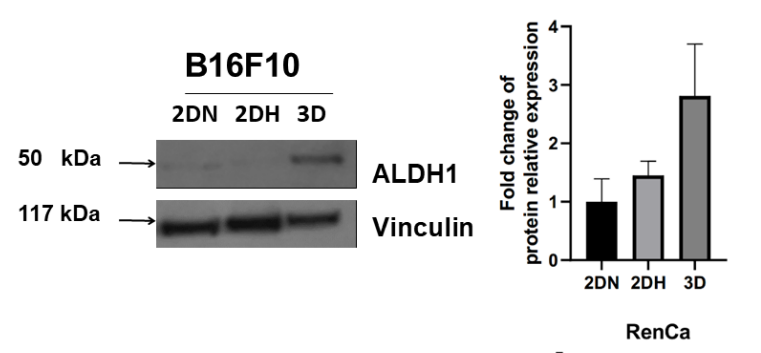

C

\section{D vs 2DH}
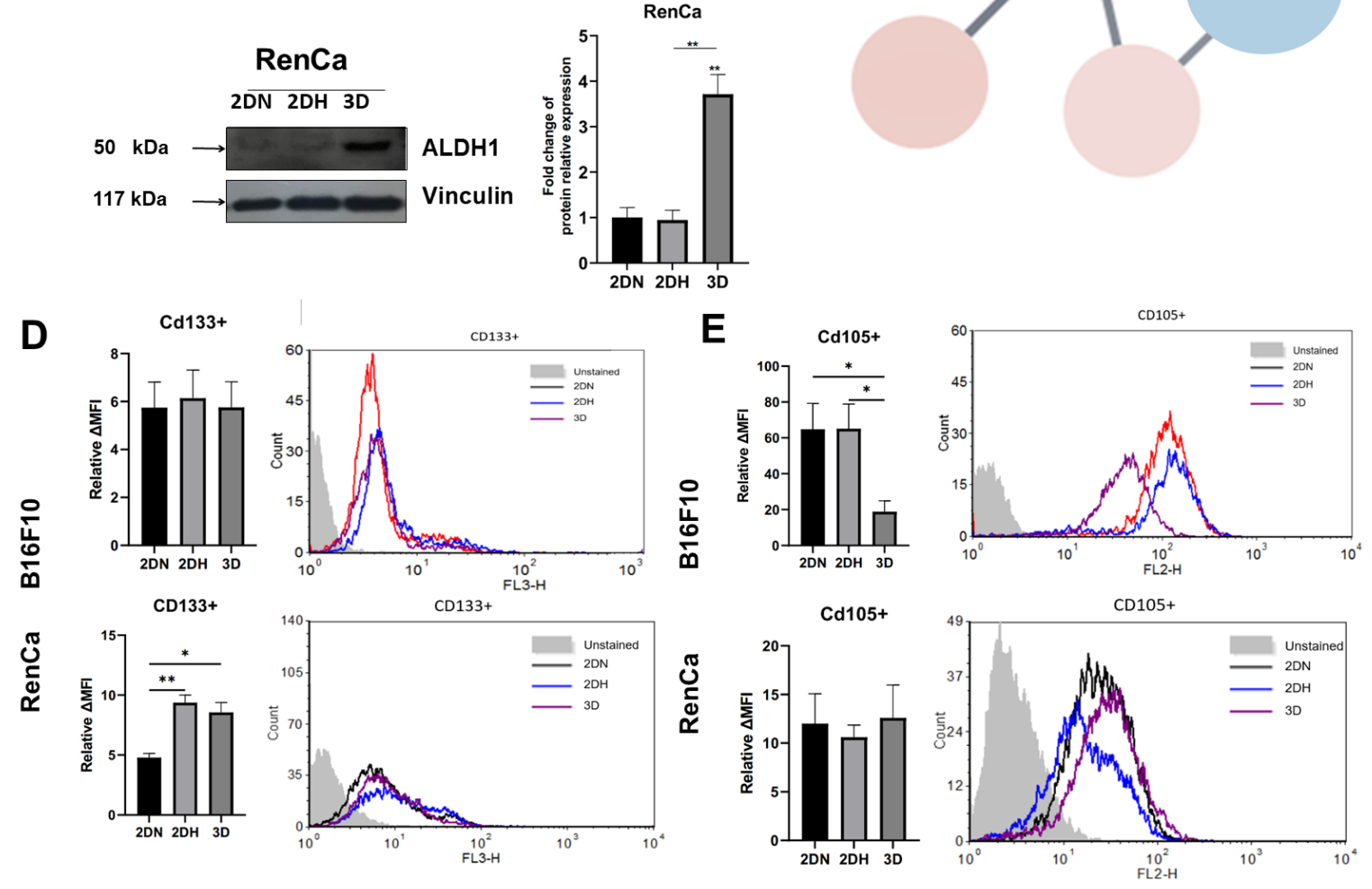

\section{Figure 4}

Effect of the culture conditions on regulation of CSC. A - ALDH1 activity determined using flow cytometry method; $\mathbf{B}$ - protein expression of ALDH1 assessed by Western blot method; $\mathbf{C}$ - Protein-protein interaction scaffold networks of ALDH2 determined by Next Generation Sequencing ( $q$-value $<0.05$ and $\left.\| \log _{2} \mathrm{FCl}>1,5\right)$ identified in RenCa cells; Blue nodes represent proteins encoded by downregulated genes and red nodes represent proteins encoded by upregulated genes. D - evaluation of CD133 positive cells 
using flow cytometry method; $\mathbf{E}$ - evaluation of CD105 positive cells using flow cytometry method. Statistical analysis was performed by One-Way ANOVA/Tukey test or Kruskal-Wallis test/ Dunn's test ${ }^{*} \mathrm{p}<0,05, \mathrm{~N} \geq 3$.

A

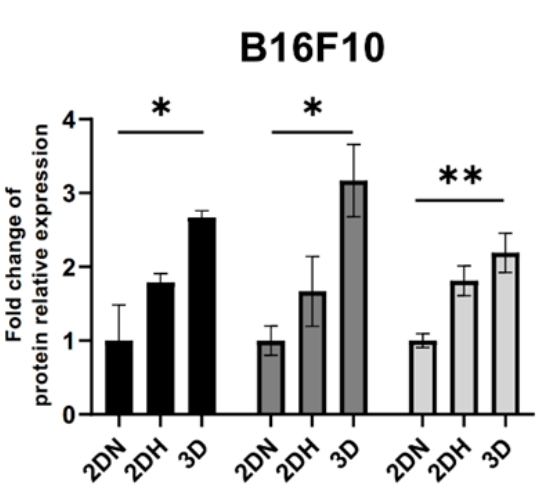

B

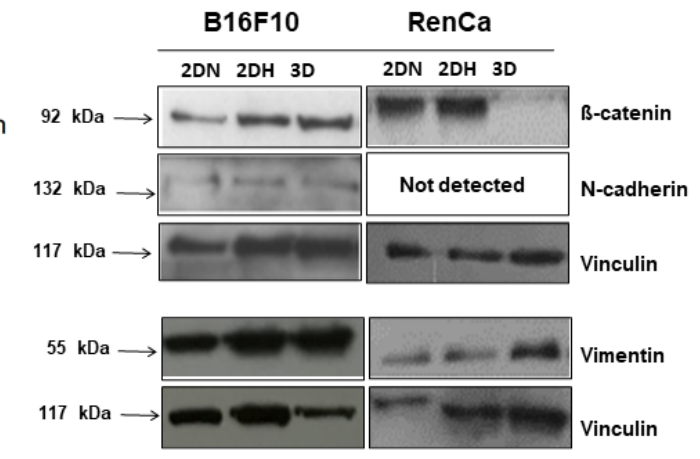

Figure 5

Relative expression of EMT markers (A, B). A - relative protein expression of B-catenin, $\mathrm{N}$-cadherin and Vimentin assessed by Western blot; Vinculin served as a quantitative loading control, B- B-catenin, Ncadherin, and Vimentin detection by Western blots. Statistical analysis was performed by One-Way ANOVA/Tukey test, ${ }^{\star} \mathrm{p}<0,05, \mathrm{~N} \geq 3$.
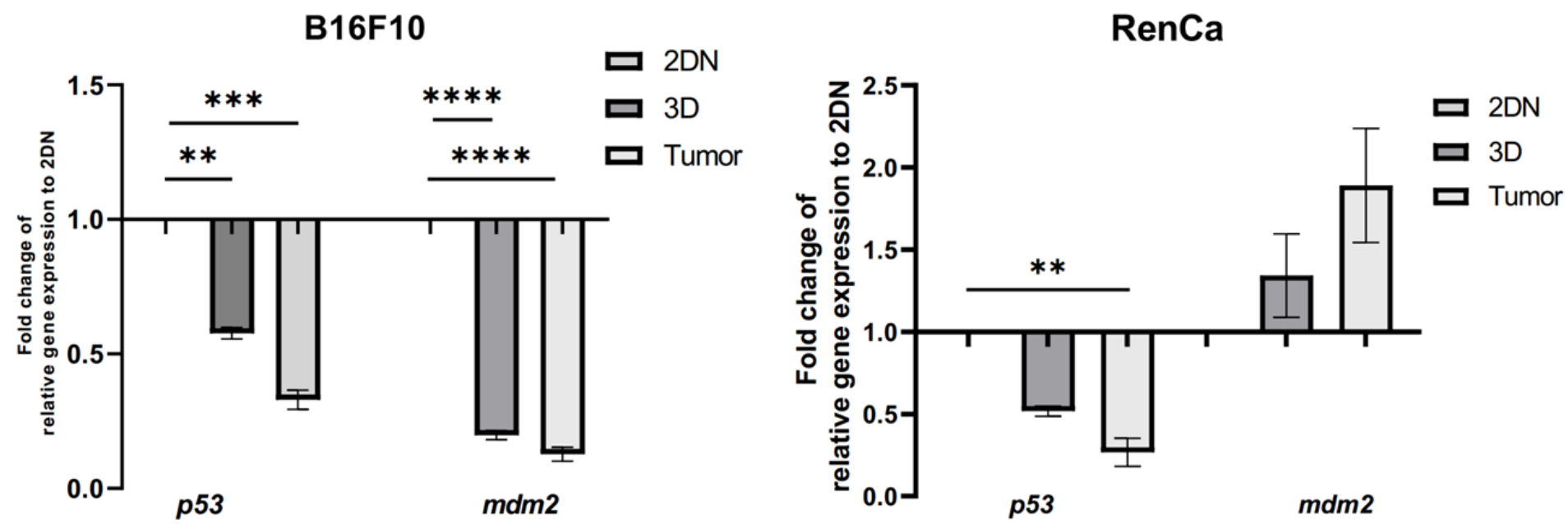

Figure 6

Comparison of proliferation capacity regulation genes. Relative expression of $m d m 2$ and $p 53$ for B16F10 and RenCa cells. All data normalized to 2DN expression. $\beta$-Actin served as a quantitative internal control. Statistical analysis was performed by One-Way ANOVA/Tukey test or Kruskal-Wallis test/ Dunn's test ${ }^{*} \mathrm{p}<0,05, \mathrm{~N} \geq 3$. 

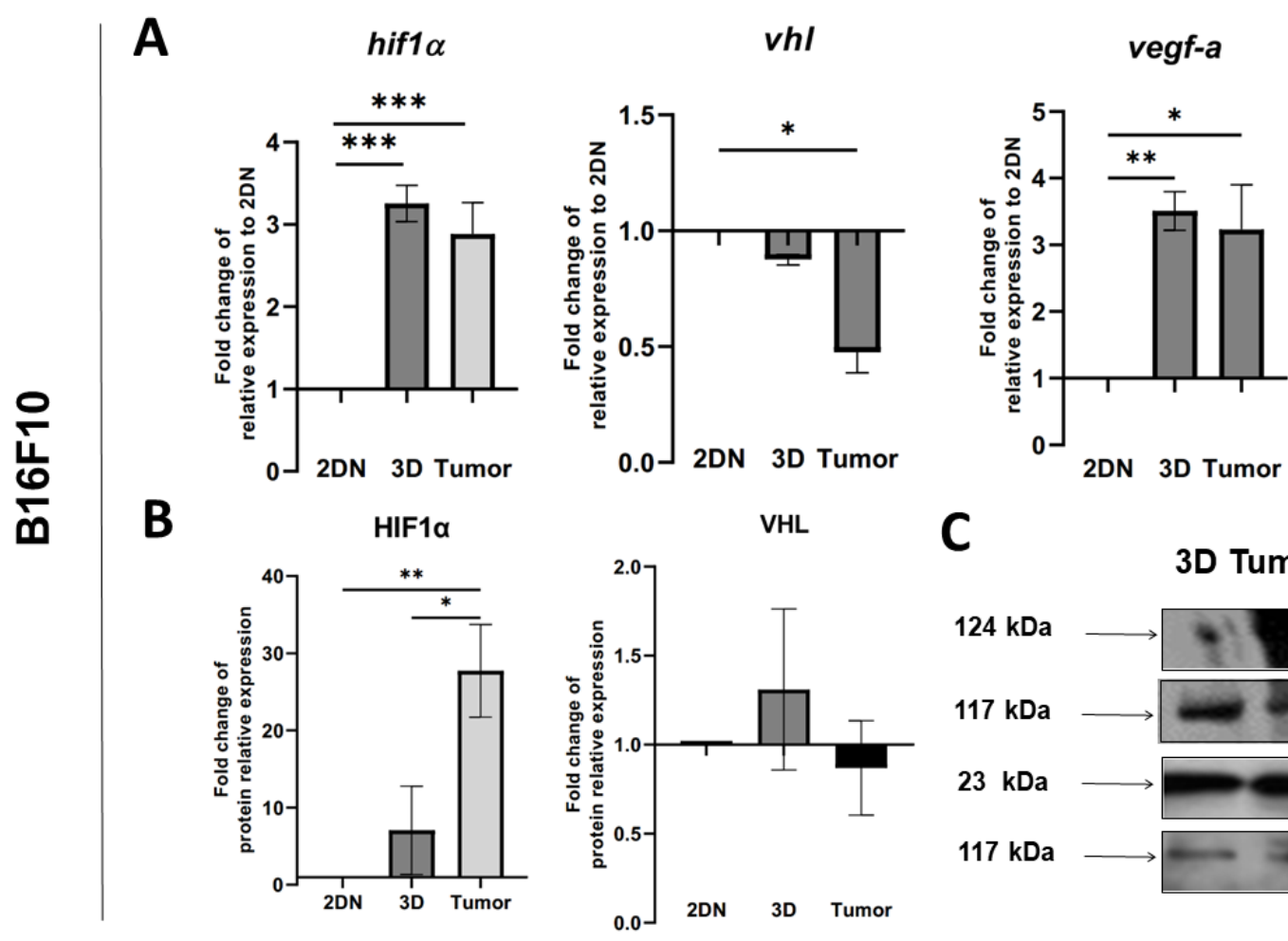

C

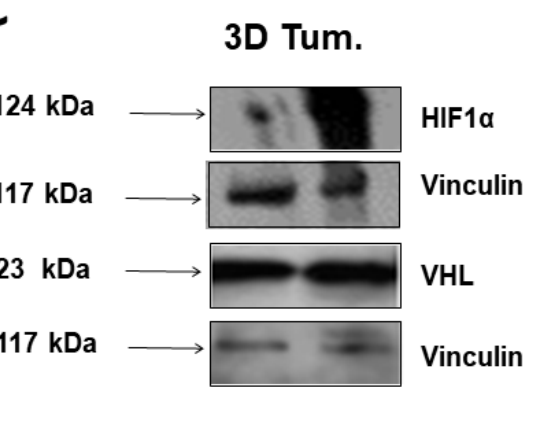

D
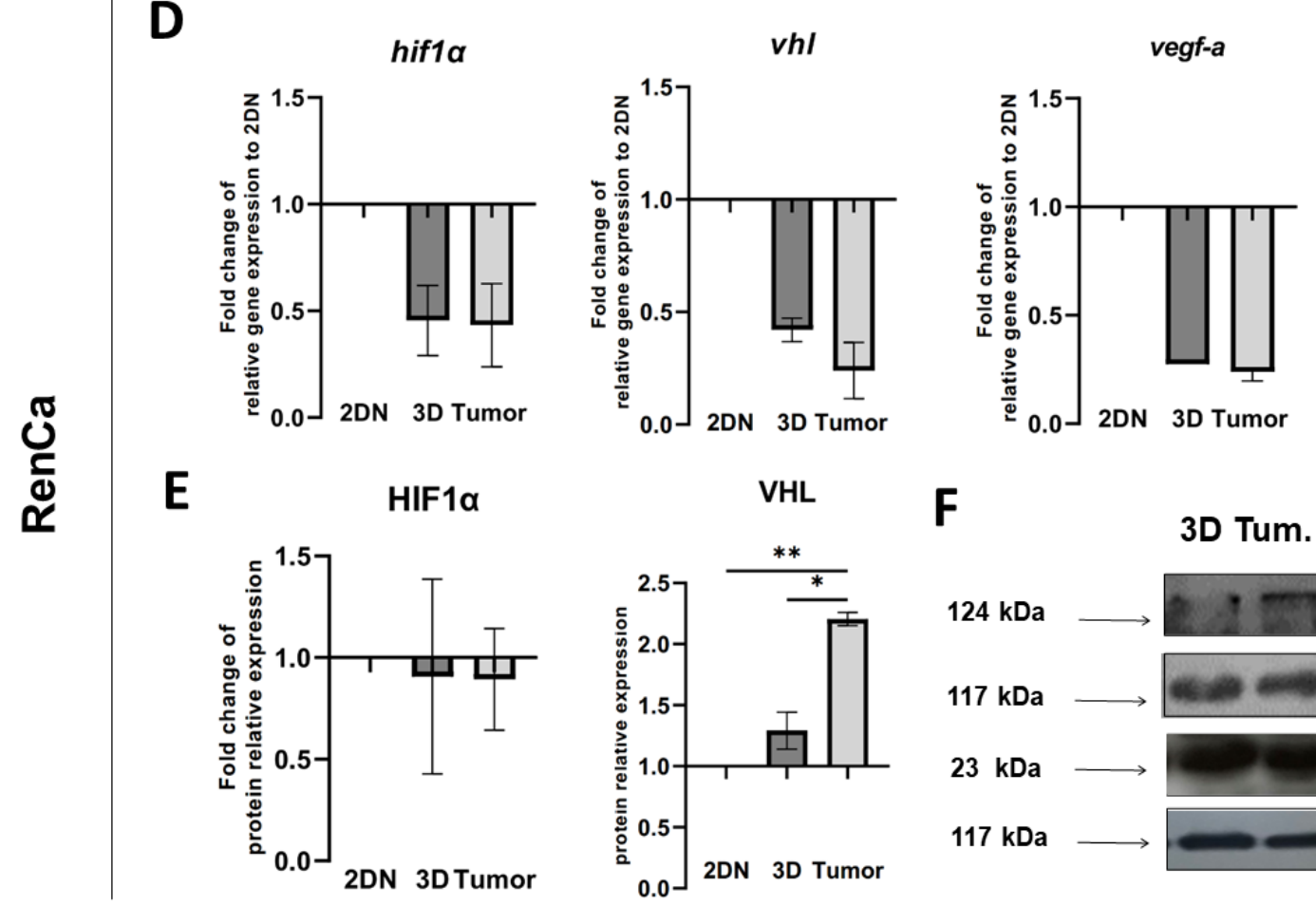

$\mathbf{F}$

3D Tum.

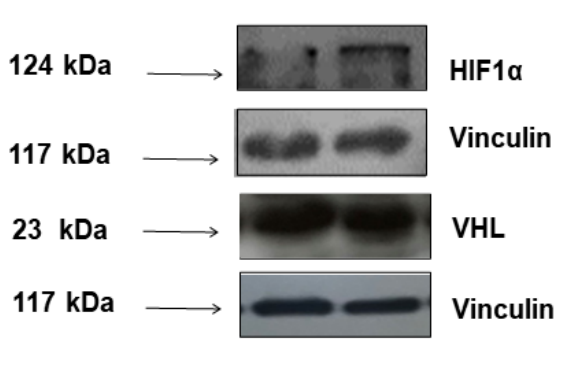

Figure 7

Comparison of the expression of hypoxia regulating genes (A, D) and proteins $(B, C, E, F)$. A, D - The gene expression of hif1a, vhl and vegf-a was determined by quantitative RT-PCR (qRT-PCR); $\beta$-actin served as a quantitative internal control. B, E - The protein expression of HIF1a, VHL and VEGF assessed by Western 
blot; Vinculin served as a quantitative loading control C, F - HIF1a and VHL detection by Western blots. Statistical analysis was performed by One-Way ANOVA/Tukey test or Kruskal-Wallis/ Dunn's test ${ }^{*} \mathrm{p}<0,05, \mathrm{~N} \geq 3$ (2DN - control).
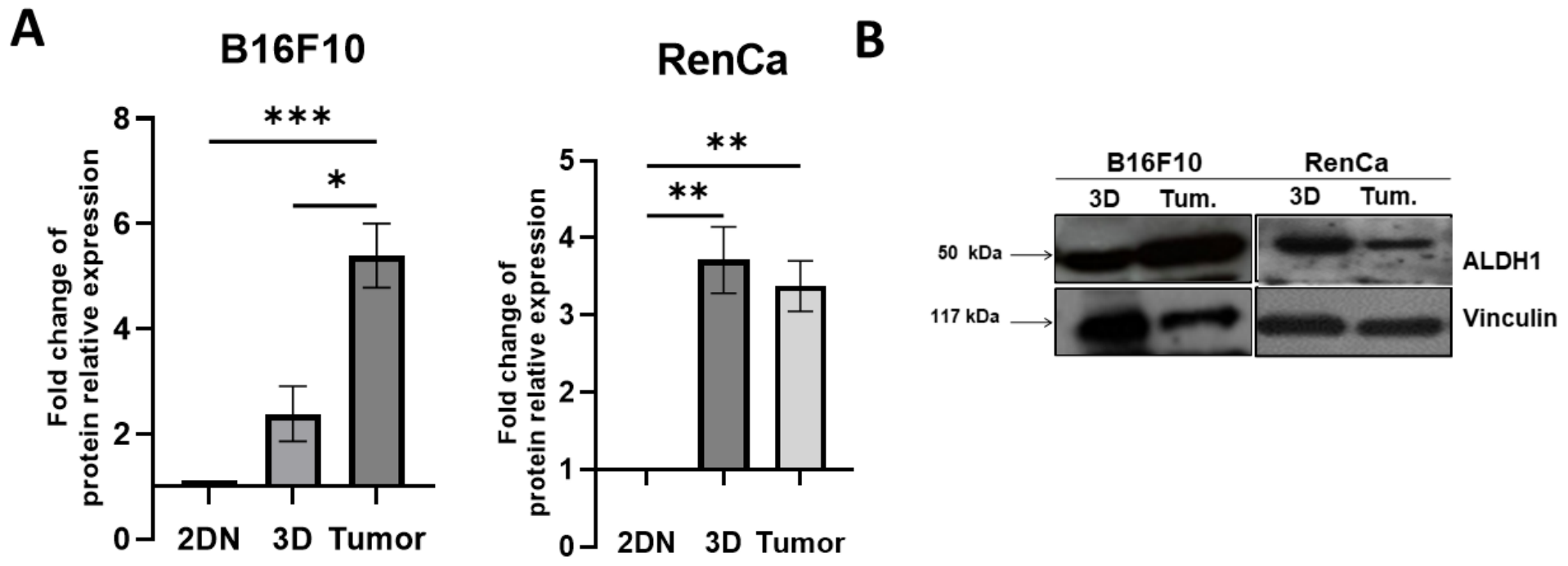

Figure 8

Comparisons of CSC expression in 3D and Tumor (A, B) A- Relative to 2DN protein expression of ALDH1, Vinculin served as a quantitative loading control. B- ALDH1 detection by Western blots. Statistical analysis was performed by One-Way ANOVA/Tukey test $p<0,05, \mathrm{~N} \geq 3$ (2DN - control).

A
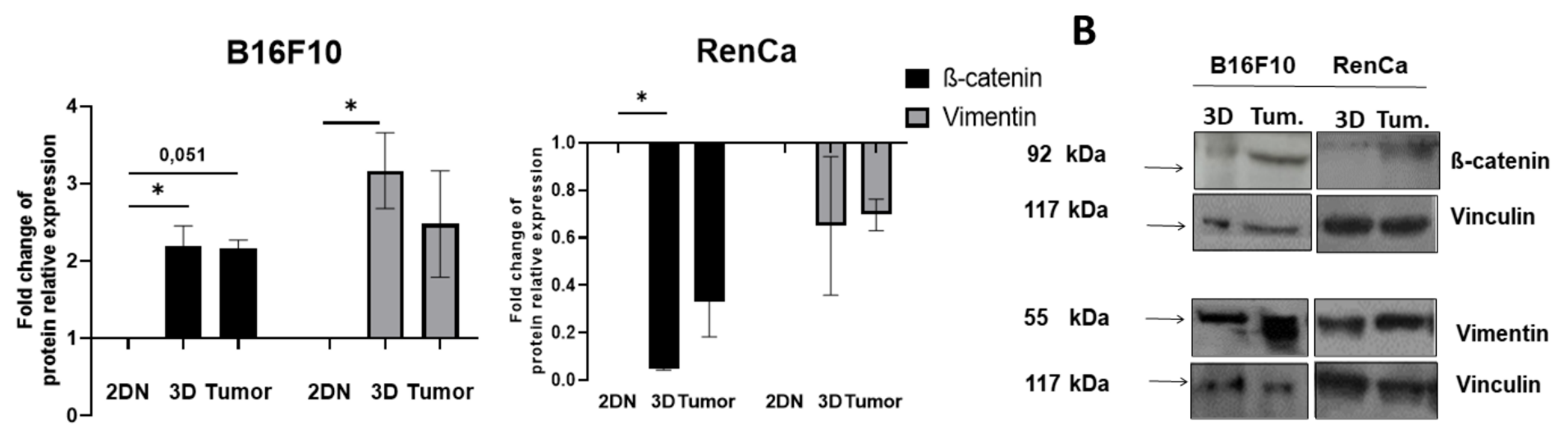

Figure 9

Comparisons of EMT markers expression in 3D and Tumor (A, B) A- Relative to 2DN protein expression of ß-catenin and Vimentin, Vinculin served as a quantitative loading control. B-Vimentin and B-catenin detection by Western blots. Statistical analysis was performed by One-Way ANOVA/Tukey test or KruskalWallis/ Dunn's test $\mathrm{p}<0,05, \mathrm{~N} \geq 3$ (2DN - control). 